\title{
Characterization of the complete chloroplast genomes of five Populus species from the western Sichuan plateau, southwest China: comparative and phylogenetic analyses
}

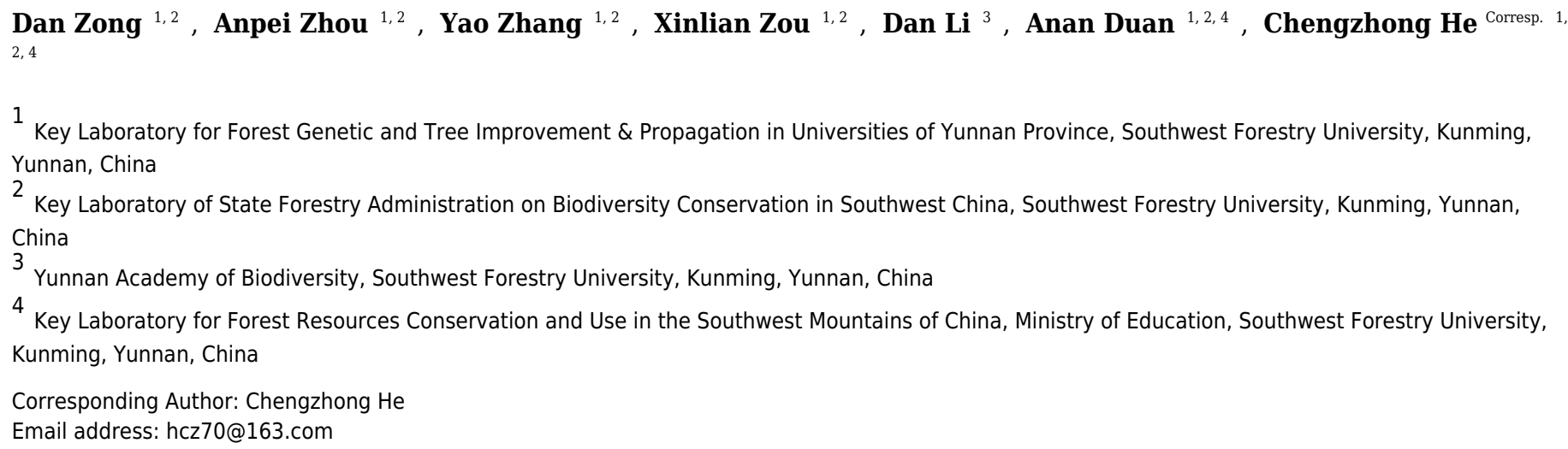

Species of the genus Populus, which is widely distributed in the northern hemisphere from subtropical to boreal forests, are among the most commercially exploited groups of forest trees. In this study, the complete chloroplast genomes of five Populus species (Populus cathayana, P. kangdingensis, P. pseudoglauca, P. schneideri and P. xiangchengensis) were compared. The chloroplast genomes of the five Populus species are very similar. The total chloroplast genome sequence lengths for the five plastomes were 156,789 bp, 156,523 bp, $156,512 \mathrm{bp}, 156,513 \mathrm{bp}$ and 156,465 bp, respectively. A total of 130 genes were identified in each genome, including 85 protein-coding genes, 37 tRNA genes and eight rRNA genes. Seven genes were duplicated in the protein-coding genes, whereas 11 genes were duplicated in the RNA genes. The GC content was $36.7 \%$ for all plastomes. We analyzed nucleotide substitutions, small inversions, SSRs and long repeats in the chloroplast genomes and found nine divergence hotspots (ccsA+ccsA-ndhD, ndhC-trnV, psbZ-trnfM, trnG-atpA, trnL-ndhJ, trnR-trnN, ycf4-cemA, ycf1, and trnR-trnN), which could be useful molecular genetic markers for future population genetic and phylogenetic studies. We also observed that two genes ( $r p o C 2$ and $r b c L$ ) were subject to positive selection. Phylogenetic analysis based on whole $\mathrm{cp}$ genomes showed that $P$. schneideri had a close relationship with $P$. kangdingensis and $P$. pseudoglauca, while $P$. xiangchengensis was a sister to $P$. cathayana. $\curvearrowright)^{\wedge} ; \mid$ MOY 


\section{Characterization of the complete chloroplast genomes of}

2 five Populus species from the western Sichuan plateau,

\section{3 southwest China: comparative and phylogenetic analyses}

4 Dan Zong ${ }^{1,2}$, Anpei Zhou ${ }^{1,2}$, Yao Zhang ${ }^{1,2}$, Xinlian Zou ${ }^{1,2}$, Dan Li ${ }^{3}$, Anan Duan ${ }^{1,2,4}$ and Chengzhong He ${ }^{1,2,4}$

$5 \quad{ }^{1}$ Key Laboratory for Forest Genetic and Tree Improvement \& Propagation in Universities of Yunnan

6 Province, Southwest Forestry University, Kunming, Yunnan, China

$7{ }^{2}$ Key Laboratory of State Forestry Administration on Biodiversity Conservation in Southwest China,

8 Southwest Forestry University, Kunming, Yunnan, China

$9{ }^{3}$ Yunnan Academy of Biodiversity, Southwest Forestry University, Kunming, Yunnan, China

$10{ }^{4}$ Key Laboratory for Forest Resources Conservation and Use in the Southwest Mountains of China,

11 Ministry of Education, Southwest Forestry University, Kunming, Yunnan, China

12

13 Corresponding Author:

14 Chengzhong He

15 Bailong Road, Kunming, Yunnan, 650224, China

Email address: Chengzhong He hecz@swfu.edu.cn 


\section{Characterization of the complete chloroplast genomes 24 of five Populus species from the western Sichuan 25 plateau, southwest China: comparative and 26 phylogenetic analyses}

27

28

29

30

31

32

Dan Zong ${ }^{1,2}$, Anpei Zhou ${ }^{1,2}$, Yao Zhang ${ }^{1,2}$, Xinlian Zou ${ }^{1,2}$, Dan $\mathrm{Li}^{3}$, Anan Duan ${ }^{1,2,4}$ and Chengzhong $\mathrm{He}^{1,2,4}$

${ }^{1}$ Key Laboratory for Forest Genetic and Tree Improvement \&Propagation in Universities of Yunnan Province, Southwest Forestry University, Kunming, China

${ }^{2}$ Key Laboratory of State Forestry Administration on Biodiversity Conservation in Southwest China, Southwest Forestry University, Kunming, China

${ }^{3}$ Yunnan Academy of Biodiversity, Southwest Forestry University, Kunming, China

${ }^{4}$ Key Laboratory for Forest Resources Conservation and Use in the Southwest Mountains of China, Ministry of Education, Southwest Forestry University, Kunming, China

Corresponding Author:

Chengzhong He

Bailong Road, Kunming, Yunnan, 650224, China

Email address: $\underline{\text { Chengzhong He hecz@swfu.edu.cn }}$

\section{Abstract}

Species of the genus Populus, which is widely distributed in the northern hemisphere from subtropical to boreal forests, are among the most commercially exploited groups of forest trees. In this study, the complete chloroplast genomes of five Populus species (Populus cathayana, $P$. kangdingensis, $P$. pseudoglauca, $P$. schneideri and $P$. xiangchengensis) were compared. The chloroplast genomes of the five Populus species are very similar. The total chloroplast genome sequence lengths for the five plastomes were 156,789 bp, 156,523 bp, 156,512 bp, 156,513 bp and $156,465 \mathrm{bp}$, respectively. A total of 130 genes were identified in each genome, including 85 protein-coding genes, 37 tRNA genes and eight rRNA genes. Seven genes were duplicated in the protein-coding genes, whereas 11 genes were duplicated in the RNA genes. The GC content was $36.7 \%$ for all plastomes. We analyzed nucleotide substitutions, small inversions, SSRs and long repeats in the chloroplast genomes and found nine divergence hotspots ( $\operatorname{cs} A+\operatorname{ccs} A-n d h D, n d h C$ trnV, psbZ-trnfM, trnG-atpA, trnL-ndhJ, trnR-trnN, ycf4-cemA, ycfl, and trnR-trnN), which could be useful molecular genetic markers for future population genetic and phylogenetic studies. We also observed that two genes ( $r p o C 2$ and $r b c L)$ were subject to positive selection.

Phylogenetic analysis based on whole cp genomes showed that P. schneideri had a close 
60

61

62

63

64

65

66

67

68

69

70

71

72

73

74

75

76

77

78

79

80

81

82

83

84

85

86

87

88

89

90

91

92

93

94

95

96

97

98

99

100

relationship with $P$. kangdingensis and $P$. pseudoglauca, while $P$. xiangchengensis was a sister to $P$. cathayana.

\section{Introduction}

The species of the genus Populus, collectively known as poplar, are widely distributed in the northern hemisphere from subtropical to boreal forests and one of the most commercially exploited groups of forest trees (Hamzeh \& Dayanandan, 2004). Because of their small genome size, fast growth rates, profuse vegetative propagation, adaptability to a variety of ecological sites, and their wood's numerous uses, Populus species have become one of the most economically important groups of forest trees and a model organism for the study of tree biology (Braatne, et al., 1992; Stettler, et al., 1996). According to a recent classification, the genus Populus is classified into six sections (Fang, et al., 1999; Zsuffa, 1975; Eckenwalder, 1996). To date, more than 100 Populus species or varieties have been reported worldwide, of which approximately 53 are endemic to China (Wan \& Zhang, 2013).

As a concentrated area of Populus resources in southwest China, the western Sichuan Plateau is dominated by mountainous and plateau geomorphology, and the mountains play a critical role in isolating plant distribution (He, et al., 2015). Meanwhile, the complex and unique natural and geographical conditions of this area provide not only diversified refuges where plants retreat in response to climatic changes but also great opportunities to develop new hybrid species (Lu, et al., 2014). However, the extensive interspecific hybridization and the high levels of morphological variation in Populus have posed great difficulties in species delimitation for systematic and comparative evolutionary studies (Hamzeh \& Dayanandan, 2004; Eckenwalder, 1996; Cronk, 2005).

Populus kangdingensis, P. pseudoglauca, P. schneideri and P. xiangchengensis are native to the western Sichuan Plateau, and they are distributed at altitudes above $3000 \mathrm{~m}$ and even above $4000 \mathrm{~m}$, whereas $P$. cathayana widely occurs in China at altitudes ranging from $800 \mathrm{~m}$ to $3000 \mathrm{~m}$. All five species overlap in the western Sichuan Plateau. Previous research has focused on their phylogenetic relationships. Liu \& Fu (2004) considered P. xiangchengensis a hybridization of $P$. schneideri and $P$. pseudoglauca based on morphological characteristics, while another study suggested that $P$. xiangchengensis was a likely hybrid species of $P$. kangdingensis and P. pseudoglauca based on morphological characteristics (Wan et al., 2009). P. schneideri was classified into section Tacamahaca. Meanwhile, it was also considered a natural hybrid formed by $P$. kangdingensis and $P$. cathayana based on ISSR (inter-simple sequence repeat) and ITS (internal transcribed spacer) molecular markers (Chen et al, 2007; Wang, 2012). P. pseudoglauca was originally classified in section Leucoides, although it was suggested to be assigned to section Tacamahaca (Zhao, 1994), and this assignment was supported by ISSR and nuclear ITS sequence markers (Wang, 2012). The relationship between the five Populus species is shown in Figure 1. All these findings suggested that the phylogenetic relationship of the five Populus species is rather complex and unclear.

Organellar DNA, which has its own genome and is inherited from one parent, is well conserved and allows for the development of informative universal markers (Howe, et al., 2003; 
101

102

103

104

105

106

107

108

109

110

111

112

113

114

115

116

117

118

119

120

121

122

123

124

125

126

127

128

129

130

131

132

133

134

135

136

137

138

139

140

Wicke, et al., 2011). The chloroplast (cp) genome, because of its relatively conserved size, gene content, structure and slow rate of nucleotide substitution within protein-coding genes, has been an ideal source of data on the phylogenetic relationships of plant taxa and their evolution and has been used to make significant contributions concerning evolutionary mechanisms for species and phylogenetic reconstruction (Khan et al., 2012; Asheesh \& Vinav, 2012; Liu et al., 2017).

With the development of sequencing technology in recent years, in addition to nuclear genome sequences, cp genes, gene spacer regions, and cp genome sequences have been widely used to study plant molecular systematics. Whole cp genomes of several species from the genus Populus have been sequenced and deposited in GenBank. Here, we compare the complete cp genomes of $P$. cathayana (GenBank accession number: MK267318), P. kangdingensis (GenBank accession number: MK267310), P. pseudoglauca (GenBank accession number:

MK267308), P. schneideri (GenBank accession number: MK267305), all sequenced in our study (Zong et al, unpublished), and P. xiangchengensis (GenBank accession number: MH910611) (Zong et al., in press). The codon usage bias, sequence divergences, mutation events, single nucleotide polymorphism (SNP) patterns and SSR distributions are compared, and a phylogenetic tree is reconstructed based on 27 complete cp genome sequences from Salicaceae. Our study provides cp genomic information for further phylogenetic reconstruction, molecular evolution research, and resources for breeding the genus Populus.

\section{Materials \& Methods}

\section{Plant materials and DNA extraction}

The fresh leaves of $P$. cathayana were collected in Kangding $\left(101^{\circ} 56^{\prime} 26^{\prime \prime} \mathrm{E}, 2^{\circ} 59^{\prime} 36^{\prime \prime} \mathrm{N}\right.$, Sichuan, China; altitude: $3109 \mathrm{~m}$ ), while the samples of $P$. kangdingensis, $P$. pseudoglauca, $P$. schneideri and $P$. xiangchengensis were collected in Kangding $\left(101^{\circ} 36^{\prime} 43^{\prime \prime} \mathrm{E}, 30^{\circ} 05^{\prime} 20^{\prime \prime} \mathrm{N}\right.$, Sichuan, China; altitude: 3554 m), Yajiang (10054'06"E, 2959'14"N, Sichuan, China; altitude: $3598 \mathrm{~m}$ ), Litang (101 $36^{\prime} 43^{\prime \prime E}, 30^{\circ} 05^{\prime} 20^{\prime \prime} \mathrm{N}$, Sichuan, China; altitude: $4018 \mathrm{~m}$ ) and Xiangcheng (9940'33"E, 285'47"N, Sichuan, China; altitude: $3530 \mathrm{~m}$ ), respectively. The voucher specimens of the five species were deposited at the herbarium of Southwest Forestry University, Kunming, China. Total genomic DNA was extracted with the Ezup plant genomic DNA prep kit (Sangon Biotech, Shanghai, China), and DNA samples were stored at $-80^{\circ} \mathrm{C}$ at the Key

Laboratory of State Forestry Administration on Biodiversity Conservation in Southwest China,

Southwest Forestry University, Kunming, China.

\section{Genome sequencing, assembly and annotation}

Total DNA was used to generate libraries with an average insert size of $400 \mathrm{bp}$, which were sequenced using the Illumina HiSeq X platform. Approximately $15.0 \mathrm{~GB}$ of raw data were generated from each genome with 150 bp paired-end read lengths. Then, the raw data were used to assemble the complete cp genome using GetOrganelle software (Jin et al., 2018) with $P$. trichocarpa as the reference. Genome annotation was performed with the program Geneious R8 (Biomatters Ltd, Auckland, New Zealand) by comparing the sequences with the cp genome of $P$. 
141 trichocarpa. The tRNA genes were further confirmed through online tRNAscan-SE web servers

142 (Schattner et al., 2005). A gene map of the annotated Populus cp genome was drawn by OGdraw 143 online (Lohse, et al., 2013).

144

145

146

147

148

149

150

151

152

153

154

155

156

157

158

159

160

161

162

163

164

165

166

167

168

169

170

171

172

173

174

175

176

177

178

179

180

\section{Indices of codon usage}

As an important indicator of codon usage bias, the relative synonymous codon usage (RSCU) value is the frequency observed for a codon divided by its expected frequency (Sharp, et al., 1986; Sharp \& Li, 1987). The amino acid compositions and RSCU values of the five Populus cp genomes were calculated using the CodonW program (Peden, 1999). Because short CDSs generally result in large estimation errors for codon usage, CDSs shorter than $300 \mathrm{bp}$ in length were excluded in codon usage calculations to avoid sampling bias (Rosenberg et al., 2003). Finally, 58 CDSs from the five cp genomes were analyzed in this study.

\section{Genome comparison}

To investigate divergence in cp genomes, identity across the whole cp genomes was visualized using the mVISTA viewer in Shuffle-LAGAN mode for the five species, with the $P$. xiangchengensis genome as the reference. MAFFT version 7 software (Katoh et al., 2005) was used to align the five plastome sequences, followed by adjustment with BioEdit. To elucidate the level of sequence variation, we then performed sliding window analysis to assess the pairwise variability (Pi) over the plastomes in DnaSP version 5 software (Librado \& Rozas, 2009). The window length was set to $600 \mathrm{bp}$, and the step size was set to $200 \mathrm{bp}$. The SNP variation was detected using the "find variation" function in Geneious R8.

\section{Identification of simple sequence repeats (SSRs) and long sequence repeats}

SSRs in five Populus cp genomes were detected using MISA (Thiel et al., 2003) with the minimal repeat number set to $12,6,5,5,5$ and 5 for mono-, di-, tri-, tetra-, penta-, and hexa nucleotide sequences, respectively. We used the online REPuter software to identify and locate forward $(\mathrm{F})$, reverse $(\mathrm{R})$, complemented $(\mathrm{C})$ and palindromic $(\mathrm{P})$ repeats. The following settings for repeat identification were used: (1) Hamming distance equal to 3; (2) minimal repeat size was set to $30 \mathrm{bp}$; (3) maximum computed repeats was set to $90 \mathrm{bp}$ (Kurtz et al., 2001).

\section{Gene selective pressure analysis of five Populus plastomes}

To examine variation in the evolutionary rates of cp genes, we calculated the nonsynonymous substitution rates $(\mathrm{Ka})$, synonymous rates $(\mathrm{Ks})$, and their ratios $(\mathrm{Ka} / \mathrm{Ks})$ using model averaging in the Ka_Ks Calculator program according to the LWL85 method (Yang \& Bielawski, 2000; Zhang et al., 2006).

\section{Phylogenetic analysis}

To explore the genetic relationships among the five species of the Populus genus, a total of 17 complete cp genomes of Populus and five plastomes of Salix were obtained from GenBank, 
181

182

183

184

185

186

187

188

189

190

191

192

193

194

195

196

197

198

199

200

201

202

203

204

205

206

207

208

209

210

211

212

213

214

215

216

217

218

219

220

and Itoa orientalis and Idesia polycarpa were used as the outgroups (Table S1). To examine the phylogenetic utility of different regions, phylogenetic analyses were performed based on the following data: (1) the complete cp DNA sequences, (2) the large single copy (LSC) region, (3) the small single copy region (SSC), (4) one inverted repeat region (IR), (5) the LSC+SSC region, (6) the LSC+SSC+IR region, and (7) a set of 85 common protein coding genes. All of the datasets were aligned using MAFFT under default settings. jModelTest 2.0 (Darriba et al., 2012) was used to determine the best-fitting model for each dataset based on the Akaike information criterion (AIC). A maximum likelihood method for phylogenetic analysis was performed based on the GTR $+\mathrm{I}+\mathrm{G}$ model in RAxML version 8 (Stamatakis, 2014).

\section{Results}

\section{Features of the five Populus plastomes}

The complete cp genomes of the five Populus species ranged from 156,465 bp ( $P$. xiangchengensis) to $156,789 \mathrm{bp}(P$. cathayana) in length. The plastome size of $P$. schneideri was only $1 \mathrm{bp}$ larger than that of $P$. pseudoglauca. The plastome size of $P$. kangdingensis was $11 \mathrm{bp}$ larger than that of $P$. pseudoglauca and $166 \mathrm{bp}$ smaller than that of $P$. cathayana (Table 1 and Fig. 2). The five cp genomes included a pair of inverted repeats (IRs) of 27,620 bp in the three species $P$. kangdingensis, $P$. pseudoglauca and $P$. schneideri and an IR pair of 27,672 bp in $P$. cathayana and 27,570 bp in $P$. xiangchengensis. The GC contents were consistent in $P$.

kangdingensis, $P$. pseudoglauca and $P$. schneideri, with $34.5 \%, 30.5 \%$ and $42.0 \%$ in the large single copy (LSC), smallshort single copy (SSC) and IR regions, respectively (Tables 1 and 2).

Each of the $P$. cathayana, $P$. kangdingensis, $P$. pseudoglauca, $P$. schneideri and $P$. xiangchengensis cp genomes encoded 130 functional genes; 112 of these were unique genes, including 78 protein-coding genes, 30 tRNA genes and 4 rRNA genes (Table S2). Most of these genes occurred as a single copy, while 18 genes were double copies: seven protein-coding genes, seven tRNA genes and four rRNA genes. The LSC region contained 59 protein-coding genes and 22 tRNA genes, whereas the SSC region contained 10 protein-coding genes and one tRNA gene.

\section{Codon usage}

Most protein-coding genes had the standard AUG sequence as the start codon, but $n d h D$ started with GUG, and rpll 6 started with ATC. ATC as an initiation codon has been reported in other cp genomes (Raubeson et al., 2007; Wu et al., 2017). GUG start codons have been reported in tobacco, but they are very rare in eukaryotic genomes (Kuroda et al., 2007). When GUG was the start codon of a protein, it was still translated as Met because of the separate tRNA used for initiation. Furthermore, the codon usage patterns of the 58 distinct protein-coding genes in the five plastomes were examined, and the plastomes of $P$. kangdingensis, $P$. pseudoglauca, and $P$. schneideri were consistent, with a length of 75,990 bp and encoding 25,330 codons, while those of $P$. cathayana and $P$. xiangchengensis were 75,864 bp and 75,840 bp in size and encoded 25,288 and 25,280 codons, respectively, as presented in Table S3. Coding ending with A and T/U had RSCU values $>1$ for the five Populus cp genomes, indicating that they were used more 
221 frequently than synonymous codons and may play major roles in the $\mathrm{A}+\mathrm{T}$ bias of entire $\mathrm{cp}$

222

223

224

225

226

227

228

229

230

231

232

233

234

235

236

237

238

239

240

241

242

243

244

245

246

247

248

249

250

251

252

253

254

255

256

257

258

259

genomes. There was a general excess of A- and U-ending codons. All three stop codons were present, with UAA being the most frequently used among the five plastomes (Table S3). In addition, leucine (Leu, 10.67\%, 10.65\%, 10.65\%, 10.65\% and 10.65\%) and cysteine (Cys, 1.14\%) were the most and least commonly coded amino acids, respectively, among the five plastomes (Table S3 and Fig. 3).

\section{Comparative analysis of the five Populus plastomes}

In this study, the cp genomes of the five Populus species were well conserved, and no gene organization rearrangement occurred when $P$. xiangchengensis was used as a reference (Fig. 4 and Fig. 5). LSC, SSC and IR sections of the three Populus species of P. kangdingensis, $P$. pseudoglauca, and P. schneideri were highly conserved and smaller than those of $P$. cathayana, while the IR regions were larger than those of $P$. xiangchengensis. Detailed comparisons of the IR-SSC and IR-LSC boundaries among the cp genomes of the five species are presented in Figure 6. Two complete or fragmented copies of $r p l 22$ and $y c f 1$ were located at the boundaries between the LSC or SSC regions and IR regions among the five Populus plastomes. The rpl22 gene crossed the IR-LSC with only $1 \mathrm{bp}$ variation in sequence length among the five plastomes. Parts of the $y c f 1$ gene (15 (P. cathayana) -158 bp (P. kangdingensis, P. pseudoglauca, and $P$. schneideri)) were found in the SSC region at the IRb-SSC junction, whereas a portion of the $y c f 1$ gene (1689 bp (P. xiangchengensis) to $1707 \mathrm{bp}$ (P. cathayana)) was present in both IRs. A $61 \mathrm{bp}$ overlap between $y c f l$ and $n d h F$ was found in P. kangdingensis, P. pseudoglauca, and $P$.

schneideri. The Pi values within the slide window of $600 \mathrm{bp}$ in the five plastomes varied from 0.00001 to 0.00335 (Table 3 ), with a mean of 0.00210 . However, nine highly variable loci (Pi>0.01), including trnG-atpA, psbZ-trnfM, trnL-ndhJ, ndhC-trnV, ycf4-cemA, trnN-trnR, ycfl, $\operatorname{ccs} A+\operatorname{ccs} A-n d h D$ and $\operatorname{trnR}$-trnN, were located in the five Populus plastomes (Fig. 7). Among these regions, $\operatorname{trn} G$-atpA, psbZ-trnfM, trnL-ndhJ, $n d h C$-trnV and $y c f 4$-cemA were located in the LSC region, $y c f 1$ and $\operatorname{css} A+\operatorname{css} A-n d h D$ were in the SSC region, and $\operatorname{trn} N$-trnR and trnR-trnN were in the IR regions.

\section{Number and forms of mutations}

We investigated SNPs, the most abundant type of mutation, in the five plastomes, with $P$. xiangchengensis as the reference. In gene-coding regions, we detected 70 SNPs in the comparative combination of $P$. cathayana- $P$. xiangchengensis, including 33 transition (Ts) and 37 transversion (Tv) SNPs, as well as 160 (97 Ts and 63 Tv), 166 (101 Ts and 65 Tv) and 164 $(99 \mathrm{Ts}$ and $65 \mathrm{Tv}$ ) SNPs in the plastomes of $P$. kangdingensis-P. xiangchengensis, $P$. pseudoglauca- $P$. xiangchengensis and $P$. schneideri-P. xiangchengensis, respectively (Table 4). Furthermore, 106 (38 Ts and $68 \mathrm{Tv}$ ), 323 (130 Ts and $193 \mathrm{Tv}$ ), 316 (130 Ts and $186 \mathrm{Tv}$ ) and 314 (131 Ts and $183 \mathrm{Tv}$ ) SNPs were detected in noncoding regions among the four comparative combinations, respectively (Table S4). 
260

261

262

263

264

265

266

267

268

269

270

271

272

273

274

275

276

277

278

279

280

281

282

283

284

285

286

287

288

289

290

291

292

293

294

295

296

297

298

299

In our study, a total of six small inversions (petA-psbJ, ndhC-trnV, trnN-trnR, $\operatorname{ccs} A-n d h D$, $n d h D-p s a C$ and $n d h F-t r n L)$ were identified based on the sequence alignment of the five complete cp genomes (Fig. 8). The small inversions from $n d h C$-trn $V$ and $n d h D$-psaC occurred in only P. xiangchengensis, those from ndhF-trnL occurred in P. pseudoglauca and $P$. schneideri, those from trnN-trnR occurred in P. kangdingensis, $P$. pseudoglauca and $P$. schneideri, and those from $\operatorname{cs} A-n d h D$ occurred in the four species other than $P$. cathayana, while the inversion from petA-psbJ occurred in the four species other than P. xiangchengensis (Fig. 8).

\section{Synonymous (Ks) and nonsynonymous (Ka) substitution rate analysis}

In this study, ratios of nonsynonymous $(\mathrm{Ka})$ versus synonymous $(\mathrm{Ks})$ substitutions were calculated for 78 shared unique protein coding genes in $P$. cathayana, $P$. kangdingensis, $P$. pseudoglauca and $P$. schneideri, with $P$. xiangchengensis as the reference. Among these genes, only 19 protein-coding genes had Ka/Ks values (Fig. 9 and Table S5). The Ka/Ks values of the remaining protein-coding genes could not be calculated because $\mathrm{Ka}$ or $\mathrm{Ks}$ was equal to 0 , indicating that these sequences were conserved without nonsynonymous or synonymous nucleotide substitution. The $\mathrm{Ka} / \mathrm{Ks}$ ratios of all genes except rpoC2 in P. pseudoglauca (1.00903) and the rbcL gene in $P$. kangdingensis (2.26407), P. pseudoglauca (2.26407) and P. schneideri (2.26407) were less than 1 (Fig. 9).

\section{SSR and long repeat analysis}

With MISA, a total of 170 SSR loci were detected, of which mononucleotide repeats (P1) comprised $148(87.06 \%)$ of all SSRs and all of the mononucleotides composed of poly A (polycytosine) and poly $\mathrm{T}$ (polythymine) repeats (Table 5). Within the five plastomes, SSR loci were primarily located in the LSC region, followed by the SSC region. A total of $15 \mathrm{SSR}$ loci were detected in the protein-coding genes $r p o B, r p o C 2$ and $r p s 8$, with all others situated in intergenic spacers and introns (Table S6). A total of 28, 39, 39, 39 and 25 SSR loci were detected in the $P$. cathayana, $P$. kangdingensis, $P$. pseudoglauca, $P$. schneideri and $P$. xiangchengensis $\mathrm{cp}$ genomes, respectively (Table 5). The corresponding numbers of these repeats in $P$. kangdingensis, P. pseudoglauca and P. schneideri matched each other and consisted of $33 \mathrm{P} 1$, two dinucleotide (P2) and four compound (C) repeats. Comparison among the five plastomes revealed that four P1 loci were found in only P.xiangchengensis, one $\mathrm{C}$ locus was found only in $P$. cathayana, and 15 SSR loci (11 P1, 1 P2 and 3C repeats) were detected in the plastomes of $P$. kangdingensis, P. pseudoglauca and P. schneideri (Table S6).

In the plastomes of the five Populus species, we found 58 repeats in $P$. cathayana, which was a higher number than those found in the other four species $(49,48,48$ and 48 repeats, respectively) (Fig. 10A). P. pseudoglauca and P. schneideri shared the same number and types of repeats (17 forward repeats $(\mathrm{F}), 23$ palindromic repeats $(\mathrm{P}), 5$ reverse repeats $(\mathrm{R})$ and 3 complement repeats (C)) (Fig. 10A). The majority of repeats $(84.86 \%)$ varied from 30 to $39 \mathrm{bp}$ in length (Fig. 10B). Variation in the number of repeat sequences situated in the four parts of the plastome was observed between species (Table S7). 
300

301

302

303

304

305

306

307

308

309

310

311

312

313

314

315

316

317

318

319

320

321

322

323

324

325

326

327

328

329

330

331

332

333

334

335

336

337

338

339

\section{Phylogenetic analysis based on the cp genome}

Seven data partitions (complete cp genomes, LSC, SSC, IR, LSC+SSC, LSC+SSC+IR region and protein coding regions) from 22 Salicaceae $\mathrm{cp}$ genomes were used to construct phylogenetic trees (Figures S1-S6 and Fig. 11). However, the best resolution in phylogenetic relationships was achieved using full cp genome sequences; thus, we discuss phylogenetic relationships mainly based on Figure 11. All of Populus was divided into four main highly supported clades (Fig. 11). Three species of the section Turanga were clade I members. Clade II included seven species ( $P$. adenopoda, $P$. alba, $P$. davidiana, $P$. qiongdaoensis, $P$. rotundifolia, $P$. tremula, and P. tremula $\times$ alba) in section Populus and one species in section Aigeiros $(P$. nigra). Clade III consisted of three species in section Tacamahaca (P. kangdingensis, $P$. schneideri and P. yunnanensis) and two species in section Leucoides (P. lasiocarpa and $P$. pseudoglauca). Clade IV included the four species in section Tacamahaca (P. balsamifera, $P$. cathayana, $P$. trichocarpa and $P$. xiangchengensis), one species in section Aigeiros ( $P$. fremontii) and one species in section Leucoides ( $P$. wilsonii). Our results showed that $P$. kangdingensis, $P$. pseudoglauca, and $P$. schneideri were in clade III, while $P$. xiangchengensis formed a sister relationship with $100 \%$ bootstrap support to $P$. cathayana in clade IV.

\section{Discussion}

In the present study, we compared five Populus plastomes, all of which assembled into single, circular, double-stranded DNA sequences presenting a typical quadripartite structure with a length of 156,465 bp to 156,789 bp, similar to most Populus cp genomes (Wang et al, 2016; Zhang \& Gao, 2016; Zheng et al, 2016; Han et al., 2017). The GC contents in the LSC, SSC and IR regions were consistent among the three species $P$. kangdingensis, $P$. pseudoglauca and $P$. schneideri, and the high GC content in the IR regions was possibly due to the presence of four ribosomal RNA sequences in these regions (Yang et al., 2016). In addition, LSC, SSC and IR sections of the three Populus species were highly conserved and smaller than those of $P$. cathayana, while the IR regions were larger than those of $P$. xiangchengensis. IRs are the most conserved regions of the $\mathrm{cp}$ genome, and the construction and expansion of IR borders are common evolutionary events and the major reasons for size differences between cp genomes (Shen et al., 2017; Wu et al., 2018).

The whole aligned sequences revealed surprisingly low divergence; however, nine regions (trnG-atpA, psbZ-trnfM, trnL-ndhJ, ndhC-trnV, ycf4-cemA, trnN-trnR, ycfl, ccs A+ccsA-ndhD and $\operatorname{trn} R-\operatorname{trn} N$ ) displayed high variation. Further work is still necessary to determine whether these nine variable loci could be used in phylogenetic analyses of related Populus species or as potential molecular markers for population genetics and phylogenetics.

Understanding nucleotide substitution rates is of fundamental importance in molecular evolution (Muse \& Gaut, 1994). During the process of searching for SNPs, we found that the cp genome sequences of $P$. kangdingensis, $P$. schneideri and $P$. pseudoglauca had similar numbers of mutations, while $P$. cathayana had a smaller number of mutations relative to $P$. 
340

341

342

343

344

345

346

347

348

349

350

351

352

353

354

355

356

357

358

359

360

361

362

363

364

365

366

367

368

369

370

371

372

373

374

375

376

377

378

379

xiangchengensis. Therefore, the phylogenetic relationships of these five species may be affected by different mutation modes (Yang et al., 2016). Furthermore, we found that the numbers and types of SSRs and long repeats of the three species $P$. kangdingensis, $P$. schneideri and $P$. pseudoglauca were basically identical. These SSR repeats and long repeats could be used to examine genetic structure, diversity, differentiation and relative species in future studies.

The Ka and Ks nucleotide substitution patterns are very important markers in gene evolution studies (Kimura, 1979). The Ka/Ks ratio is indicative of changes in selective pressures. $\mathrm{Ka} / \mathrm{Ks}$ values $>1,=1$, and $<1$ indicate positive selection, natural evolution and purifying selection affecting the coding portions, respectively (Sharp \& Li, 1987; Yang \& Bielawski, 2000; Lawrie et al., 2013). However, the $\mathrm{Ka} / \mathrm{Ks}$ ratio is less than one in most protein-coding regions (Makalowski \& Boguski, 1998). When we compared the 78 common unique protein coding genes, the $\mathrm{Ka} / \mathrm{Ks}$ ratios were less than one, except the ratio for the gene $r p o C 2$ in one pairwise comparison of $P$. pseudoglauca-P. xiangchengensis and the ratio for the gene $r b c L$ in the pairwise comparisons of $P$. kangdingensis- $P$. xiangchengensis, $P$. pseudoglauca- $P$.

xiangchengensis and $P$. schneideri-P. xiangchengensis, indicating that these two genes are undergoing positive selection and that at least some of the mutations concerned must be advantageous.

Small inversions in the cp genome of angiosperms are ubiquitous and commonly associated with a hairpin secondary structure (Kim \& Lee, 2005; Catalano et al., 2009). A distinctive feature of these inversions is that they are flanked by inverted repeats that range from 8 to $50 \mathrm{bp}$, such that the inverted repeats form the stem and the segment between them forms the loop (Catalano et al., 2009). These small inversions are generally recognized by pairwise comparisons between sequences. In our study, six small inversions were discovered based on the sequence alignment of the five complete cp genomes. Two small inversions from $n d h C$-trnV and $n d h D$-psaC were only present in P. xiangchengensis, while three from ccs $A$ - $n d h D, n d h F-\operatorname{trn} L$ and trnN-trnR were shared by P. pseudoglauca and P. schneideri. These small inversion regions will provide abundant information for marker development in phylogenetic analyses of related Populus species. Small inversions in the $c c s A-n d h D$ and petA-psbJ intergenic regions have been reported in other studies (Song et al., 2015; 2016; Dong et al., 2017). However, small inversions of noncoding sequences may influence sequence alignment and character interpretation in phylogeny reconstructions, so caution is necessary when using cp noncoding sequences for phylogenetic analysis.

The cp genome is widely employed to study evolution through phylogenetics, and it has been suggested to be useful for phylogenetic reconstruction at low taxonomic levels (Zhang et al., 2011; Ma et al., 2014; Yang et al., 2014; Zhang et al., 2016). It has also been postulated to be a potential ultrabarcode or organelle-scale barcode for taxonomically complex groups (Kane et al., 2012). The key interest of the current study is to resolve previous phylogenetic controversies in Populus (Zhao, 1994; Liu \& Fu, 2004; Chen et al., 2007; Wan et al., 2009; Wang, 2012) by using complete cp genome sequences. The results revealed that $P$. nigra (section Aigeiros) was nested among members of the section Populus, which is supported by previous studies (Rajora 
380

381

382

383

384

385

386

387

388

389

390

391

392

393

394

395

396

397

398

399

400

401

402

403

404

405

406

407

408

409

410

411

412

413

414

415

416

417

418

419

\& Dancik, 1995; Hamzeh \& Dayanandan, 2004). Both previous studies found that P. nigra showed higher similarities to $P$. alba than to other species.

The position of $P$. pseudoglauca confirms the previously published phylogeny described by Chao \& Liu (1991), in which P. pseudoglauca was classified into section Tacamahaca according to fossil evidence, paleogeography, paleoclimate, and modern distribution. The species $P$.

schneideri, which is distributed in the western Sichuan Plateau at altitudes of 3000 to $4000 \mathrm{~m}$, has remained a topic of debate among scientists. According to its morphology, it is similar to $P$. cathayana (Fang et al., 1999). Wan et al. (2013) suggested that $P$. schneideri is generally closer to $P$. cathayana than $P$. kangdingensis, and it is a natural hybrid between the ancestors of $P$. cathayana and $P$. kangdingensis based on cpDNA and nuclear DNA sequence data as well as amplified restriction fragment polymorphism (AFLP) analyses. Other studies considered $P$. schneideri to be a variety of $P$. kangdingensis based on morphological traits (Chao \& Liu, 1991; Yu et al., 2003; Liu \& Fu, 2004). Chen et al. (2007) suggest that P. schneideri is generally more highly related to $P$. kangdingensis than to $P$. cathayana based on cpSSR analysis. Our data revealed that $P$. schneideri had a close relationship with $P$. pseudoglauca and $P$. kangdingensis. $P$. schneideri and $P$. kangdingensis are both unique to the western Sichuan Plateau, and they share similar altitude and habitat requirements (Yu et al., 2003). In addition, the plastome characteristics, SNP variations, SSR and long repeats and small inversions all showed that $P$. kangdingensis, $P$. pseudoglauca and $P$. schneideri had similar structure and variation models. Therefore, we speculated that these three species may be the same species; however, more population data are needed for further analyses. P. xiangchengensis was a sister to $P$. cathayana, as revealed by cp genome sequence analysis, which did not support the viewpoint that $P$. xiangchengensis is a natural hybrid species of either $P$. schneideri and $P$. pseudoglauca or $P$. kangdingensis and $P$. pseudoglauca. It is our hope that the five plastomes will provide useful resources for better understanding the phylogeny and relationships of the genus Populus.

\section{Conclusions}

This study reports the comparative analysis of five Populus cp genome sequences with detailed gene annotation. Comparing the five plastomes showed that the plastomes were similar in structure and had a high degree of synteny. Nine divergent regions (trnG-atpA, psbZ-trnfM, $\operatorname{trnL} L-n d h J, n d h C$-trnV $, y c f 4-c e m A, \operatorname{trn} N$-trnR, $y c f 1, \operatorname{cs} A+\operatorname{css} A-n d h D$ and trnR-trnN) were identified and may be utilized as potential molecular markers for population genetic and phylogenetic studies in Populus. Furthermore, among the five cp genomes, $P$. kangdingensis, $P$. pseudoglauca and $P$. schneideri showed little difference in their SNP loci and SSRs. The results of the phylogenetic analyses showed that $P$. schneideri had the closest affinity to $P$. kangdingensis and was a sister to $P$. pseudoglauca, while $P$. cathayana had a close relationship with $P$. xiangchengensis. The characterization of these five plastomes will provide useful resources for better understanding the phylogeny and relationships of the genus Populus.

\section{Acknowledgements}


420

421

422

423

424

425

426

427

428

429

430

431

432

433

434

435

436

437

438

439

440

441

442

443

444

445

446

447

448

449

450

451

452

453

454

455

456

457

458

459

We would like to thank Prof. Wuyuan Yin for his help with sample collection and species identification. We are grateful to Dr. Hui Zhan for language review. We also thank the editor and the two anonymous reviewers for their constructive comments, which helped us improve this manuscript.

\section{References}

Asheesh S, Vinay S. 2012. Evolutionary analysis of plants using chloroplast. German: LAP Lambert Academic Publishing.

Braatne JH, Hinckly TM, Stettler, RF. 1992. Nuclear ribosomal DNA phylogeny water supply on the physiological and morpgological components of plant water balance in Populus trichocarpa, Populus deltoids and their F1 hybrids. Tree physiology 11: 325-340.

Catalano SA, Saidman BO, Vilardi JC. 2009. Evolution of small inversions in chloroplast genome: a case study from a recurrent inversion in angiosperms. Cladistics 25(1): 93-104.

Chao N, Liu J. 1991. Taxonnmic studies on Populus L. in southwestern China (I). J. Wuhan Bot. Res. 9(3): 229-238.

Chen K, Peng YH, Wang YH, Korpelainen H, Li CY. 2007. Genetic relationships among poplar species in section Tacamahaca (Populus L.) from western Sichuan, China. Plant Science 172(2): 196-203.

Cronk Q. 2005. Plant eco-devo: the potential of poplar as a model organism. New Phytologist 166(1): 39-48.

Darriba D, Taboada GL, Doallo R, Posada D. 2012. jModelTest 2: more models, new heuristics and parallel computing. Nat. Methods 9: 772.

Dong WP, Xu C, Li WQ, Xie XM, Lu YZ, Liu YL, Jin XB, Suo ZL. 2017. Phylogenetic resolution in Juglans based on complete chloroplast genomes and nuclear DNA sequences. Front. Plant Sci. 8.

Eckenwalder JE. 1996. Systematics and evolution of Populus. In Stettler RF, Bradshaw HD, Heilman PE, Hinckley TM. Biolology of Populus and its implications for management and conservation. Canada: NRC research press, 7-32.

Fang ZF, Zhao SD, Skvortsov AK. 1999. Flora of China (English version). Beijing: Science press, 4: 139-274.

Hamzeh M, Dayanandan S. 2004. Phylogeny of Populus (Salicaceae) based on nucleotide sequences of chloroplast trnT-trnF region and nuclear rDNA. American Journal of Botany 91(9): 1398-1408.

Han XM, Wang YM, Liu YJ. 2017. The complete chloroplast genome sequence of Populus wilsonii and its phylogenetic analysis. Mitochondrial DNA Part B: resources 2(2): 932-933.

He CZ, Li JM, Yun T, Zong D, Zhou AP, Ou GL, Yin WY. 2015. SRAP analysis on the effect of geographic isolation on population genetic structure of Populus davidiana in Tibetan-inhabited regions in Southwest China. Forest Research 28(2): 152-157.

Howe CJ, Barbrook AC, Koumandou VL, Nisbet RER, Symington HA, Wightman TF. 2003. Evolution of the chloroplast genome. Phil. Trans. R Soc. L and B 358(1429): 99-107. 
460

461

462

463

464

465

466

467

468

469

470

471

472

473

474

475

476

477

478

479

480

481

482

483

484

485

486

487

488

489

490

491

492

493

494

495

496

497

498

Jin JJ, Yu WB, Yang JB, Song Y, Yi TS, Li DZ. 2018. GetOrganelle: a simple and fast pipeline for de novo assemble of a complete circular chloroplast genome using genome skimming data. BioRxiv Preprint.

Kane N, Sveinsson S, Dempewolf H, Yang JY, Zhang D, Engels JM, Cronk Q. 2012. Ultrabarcoding in cacao (Theobram spp.; Malvaceae) using whole chloroplast genomes and nuclear ribosomal DNA. Am. J. Bot. 99(2): 320-329.

Katoh K, Kuma K, Toh H, Miyata T. 2005. MAFFT version 5: improvement in accuracy of multiple sequence alignment. Nucleic Acids Res. 33(2): 511-518.

Khan A, Khan I, Heinze B, Azim MK. 2012. The chloroplast genome sequence of date palm (Pheonix dactylifera L. cv. 'Aseel'). Plant Mol. Biol. Rep. 30(3): 666-678.

Kim KJ, Lee HL. 2005. Wide spread occurrence of small inversions in the chloroplast genomes of land plants. Mol. Cells 19(1): 104-113.

Kimura M. 1979. The neutral theory of molecular evolution. Sci. Am. 241: 98.

Kuroda H, Suzuki H, Kusumegi T, Hirose T, Yukawa Y, Sugiura M. 2007. Translation of $p s b C$ mRNAs starts from the downstream GUG, not the upstream AUG, and requires the extended shine-dalgarno sequence in tobacco chloroplasts. Plant Cell Physiol. 48(9): 13741378.

Kurtz S, Choudhuri JV, Ohlebusch E, Schleiermacher C, Stoyem J, Giegerich R. 2001. REPuter: the manifold applications of repeat analysis on a genomic scale. Nucleic Acids Res. 29(22): 4633-4642.

Lawrie DS, Messer PW, Hershberg R, Petrov DA. 2013. Strong purifying selection at synonymous sites in D. melanogaster. PLoS Genet. 9(5): 261-270.

Librado P, Rozas J. 2009. Dnasp v5: a software for comprehensive analysis of DNA polymorphism data. Bioinformatics 25(11): 1451-1452.

Liu LX, Li R, Worth JP, Li X, Li P, Cameron KM, Fu CX. 2017. The complete chloroplast genome of Chinese Bayberry (Morella rubra, Myricaceae): Implications for understanding the evolution of Fagales. Front. Plant Sci. 8: 968.

Liu YQ, Fu DQ. 2004. Development and utilization of sect. III Tacamahaca gene resources on the plateau of western Sichuan. Journal of Central South Forestry University 24(5): 129132.

Lohse M, Drechsel O, Kahlau S, Bock R. 2013. OrganellarGenomeDRAW-a suite of tools for generating physical maps of plastid and mitochondrial genomes and visualizing expression data sets. Nucleic Acids Res 41: 575-581.

Lu ZQ, Tian B, Liu BB, Yang C, Liu JQ. 2014. Origin of Ostryopsis intermedia (Betulaceae) in the southeast Qinghai-Tibet Plateau through hybrid speciation. Journal of Systematics and Evolution 52(3): 250-259.

Ma PF, Zhang YX, Zeng CX. Guo ZH, Li DZ. 2014. Chloroplast phylogenomic analyses resolve deep-level relationships of an intractable bamboo tribe Arundinarieae (Poaceae). Syst. Biol. 63(6): 933-950. 
499

500

501

502

503

504

505

506

507

508

509

510

511

512

513

514

515

516

517

518

519

520

521

522

523

524

525

526

527

528

529

530

531

532

533

534

535

536

537

538

Makalowski W, Boguski MS. 1998. Evolutionary parameters of the transcribed mammalian genome: an analysis of 2,820 orthologous rodent and human sequences. Proc. Natl. Sci. U.S.A. 95(16): 9407-9412.

Muse SV, Gaut BS. 1994. A likelihood approach for comparing synonymous and nonsynonymous nucleotide substitution rates, with application to the chloroplast genome. Mol. Biol. Evol. 11(5): 715-724.

Peden JF. 1999. Analysis of codon usage. PhD thesis, University of Nottingham, UK.

Rajora OP, Dancik BP. 1995. Chloroplast DNA variation in Populus II interspecific restriction fragment polymorphisms and genetic relationships among Populus deltoids, . nigra, $P$. maximowiczii, and P. × canadensis. Theor. Appl. Genet. 90(3-4): 324-330.

Raubeson LA, Peery R, Chumley TW, Dziubek C, Fourcade HM, Boore J, Jansen RK. 2007. Comparative chloroplast genomics: analyses including new sequences from the angiosperms Nuphar advena and Ranunculus macranthus. BMC Genomics. 8: 174.

Rosenberg MS, Subramanian S, Kumar S. 2003. Patterns of transitional mutation biases within and among mammalian genomes. Mol. Biol. Evol. 20(6): 988-993

Schattner P, Brooks AN, Lowe TM. 2005. The tRNAscan-SE, snoscan and snoGPS web servers for the detection of tRNAs and snoRNAs. Nucleic. Acids. Res. 33: 686-689.

Sharp PM, Li WH. 1987. The rate of synonymous substitution in enterobacterial genes is inversely related to codon usage bias. Mol. Biol. Evol. 4(3): 222-230.

Sharp PM, Tuohy TMF, Mosurski KR. 1986. Codon usage in yeast cluster analysis clearly differentiates highly and lowly expressed genes. Nucleic Acids Res. 14(13): 1281-1295.

Shen XR, Wu ML, Liao BS, Liu ZX, Bai R, Xiao SM, Li XW, Zhang BL, Xu J, Chen SL. 2017. Complete chloroplast genome sequence and phylogenetic analysis of the medicinal plant Artemisia annua. Molecules 22(8): 1330.

Song Y, Dong W, Liu B, Xu C, Yao X, Gao J, Corlet RT. 2015. Comparative analysis of complete chloroplast genome sequences of two tropical trees Machilus yunnanensis and Machilus balansae in the family Lauraceae. Front. Plant Sci. 6: 662.

Song Y, Yao X, Tan YH, Gan Y, Corlet RT. 2016. Complete chloroplast genome sequence of the avocado: gene organization, comparative analysis, and phylogenetic relationships with other Lauraceae. Can. J. For. Res. 46 (11): 1293-1301.

Stamatakis A. 2014. RAxML version 8: a tool for phylogenetic analysis and post-analysis of large phylogenies. Bioinformatics 30(9): 1312-1313.

Stettler RF, Bradshaw HD, Heilman PE, Hinckley TM. 1996. The role of hybridization in genetic manipulation of Populus. In: Biology of Populus and its implications for management and conservation. Canada : NRC Research Press.

Thiel T, Michalek W, Varshney RK. 2003. Exploiting EST databases for the development and characterization of gene-derived SSR markers in barley (Hordeum vulgare L.). Theoretical and Applied Genetics 106(3): 411-422.

Wan XQ, Zhang F. 2013. An overview of Populus genetic resources in southwest China. Forestry chronicle 89(1): 79-87. 
539

540

541

542

543

544

545

546

547

548

549

550

551

552

553

554

555

556

557

558

559

560

561

562

563

564

565

566

567

568

569

570

571

572

573

574

575

576

577

578

Wan XQ, Zhang F, Zhong Y, Ding YH, Wang LW, Hu TX. 2013. Study of genetic relationships and phylogeny of the native Populus in southwest China based on nucleotide sequences of chloroplast trnT-trnF and nuclear DNA. Plant Syst. Evol. 299(1): 57-65.

Wan XQ, Zhang F, Zhong Y, Wang CL, Ding YH, Hu TX, Zhai MP, Qian ZL. 2009. Conservation and application of the genetic resources of native poplars in southwest China. Scientia Slivae Sinicae 45(4): 139-144.

Wang MF. 2012. Analysis the genetic relationship of the native Populus in Sichuan by ISSR and ITS sequences. PhD thesis, Sichuan Agricultrual University.

Wang TJ, Fan LQ, Guo XL, Wang K. 2016. Characterization of the complete chloroplast genome of Populus qiongdaoensis T. Hong et P. Luo. Conservation Genet Resour 8(4): 1-3.

Wicke S, Schneeweiss GM, Müller KF, Quandt D. 2011. The evolution of the plastid chromosome in land plants: Gene content, gene order, gene function. Plant Mol. Biol. 76(35): 273-297.

Wu ML, Qing L, Xu J, Li XW. 2018. Complete chloroplast genome of the medicinal plant Amomum compactum: gene organization, comparative analysis, and phylogenetic relationships within Zingiberales. Chinese Medicine 13(1): 10.

Wu ML, Li Q, Hu ZG, Li XW, Chen SL. 2017. The complete Amomum Kravanh chloroplast genome sequence and phylogenetic analysis of the Commelinids. Molecules 22(11): 1875.

Yang JB, Li DZ, Li HT. 2014. Highly effective sequencing whole chloroplast genomes of angiosperms by nine novel universal primer pairs. Molecular Ecology Resources 14(5): 1024-1031.

Yang YC, Zhou T, Duan D, Yang J, Feng L, Zhao GF. 2016. Comparative analysis of the complete chloroplast genomes of five Quercus species. Front. in Plant Sci. 7: 959.

Yang ZH, Bielawski JP. 2000. Statistical methods for detecting molecular adaptation. Trends Ecol. 15(12): 496-503.

Yu SQ, Liu J, Fu DR, Liu DJ, Liu YQ. 2003. Characteristics of Tacamachaca genes in the western Sichuan Plateau. Journal of Zhejiang Forestry College 20: 27-31.

Zhang QJ, Gao LZ. 2016. The complete chloroplast genome sequence of desert poplar (Populus euphratica). Mitochondrial DNA 27(1): 721.

Zhang YJ, Du LW, Liu A, Chen JJ, Wu L, Hu WM, Zhang W, Kim K, Lee SC, Yang TJ, Wang Y. 2016. The complete chloroplast genome sequences of five Epimedium species: lights into phylogenetic and taxonomic analysis. Front. Plant Sci. 7: 306.

Zhang YJ, Ma PF, Li DZ. 2011. High-throughput sequencing of six bamboo chloroplast genomes: phylogenetic implications for temperate woody bamboos (Poaceae: Bambusoideae). PloS ONE 6(5): e20596.

Zhang Z, Li J, Zhao XQ, Wang J, Wong KSG, Yu J. 2006. KaKs_calculator: Calculating Ka and Ks through model selection and model averaging. Genomics, Proteomics \& Bioinformatics, 4(4): 259-263.

Zhao N. 1994. Taxonomic study on Salicaceae in Sichuan and its adjacent regions (Third). Sichuan Forestry Science and Technology 15: 1-11. 
579 Zheng HL, Fan LQ, Wang TJ, Zhang L, Ma T, Mao KS. 2016. The complete chloroplast

580 genome of Populus rotundifolia (Salicaceae). Conservation Genet. Resour. 8(4): 1-3.

581 Zong D, Zhou AP, Li D, He CZ. 2018. The complete chloroplast genome of Populus

582

583 xiangchengensis, an endemic species in southwest China. Mitochondrial DNA part B In

584 Zsuffa L. 1975. A summary review of interspecific breeding in the genus Populus. In

585 proceedings of the $14^{\text {th }}$ annual meeting of the Canadian tree improvement association, part

586

2. Canadian Forest Service, Ottawa, Ontario, Canada 107-123.

587

588 
589 Figure captions

590 Figure 1 The hybridization schematic diagram of five Populus species. (A) P. kangdingensis;

591 (B) P. cathayana; (C) P. schneideri; (D) P. pseudoglauca; (E) P. xiangchengensis. P.

592 schneideri $=P$. kangdingensis $\times P$. cathayana, $P$. xiangchengensis $=P$. kangdingensis $\times P$.

593 pseudoglauca, $P$. xiangchengensis $=P$. schneideri $\times$ P. pseudoglauca.

594 Figure 2 Gene map of the five Populus species cp genomes. The genes that are drawn outside

595 of the circle are transcribed clockwise, whereas those that are drawn inside the circle are

596 transcribed counterclockwise. Large single copy (LSC), small single copy (SSC) and inverted

597 repeats (IRa and IRb) are indicated.

598 Figure 3 Amino acid frequencies of the protein-coding sequences of the five plastomes 599 Figure 4 Whole chloroplast genome alignments for five Populus species using the mVISTA

600 program, with $\boldsymbol{P}$. xiangchengensis as the reference. The $\mathrm{Y}$-scale indicates identity from $50 \%$

601 to $100 \%$, Gray arrows indicate the position and direction of each gene. Red indicates non-coding

602 sequences (CNS), blue indicates the exons of protein-coding genes (exon) and line green

603 indicates the tRNA or rRNA genes.

604 Figure 5 Mauve alignment of the cp genomes of five Populus species. (A) Cp genome of $P$.

605 xiangchengensis; (B) Cp genome of $P$. cathayana; (C) Cp genome of $P$. kangdingensis; (D) Cp

606 genome of $P$. pseudoglauca; (E) Cp genome of $P$. schneideri.

607 Figure 6 Comparison of LSC, SSC and IR region borders among cp genomes of five

608 Populus species. (A) Cp genome of P. xiangchengensis; (B) Cp genome of P. cathayana; (C) Cp

609 genome of $P$. kangdingensis; (D) Cp genome of P. pseudoglauca; (E) Cp genome of $P$.

610 schneideri.

611 Figure 7 Sliding window analyses of the whole plastomes of five Populus species. The

612 genetic divergence among the plastomes of $P$. cathayana, $P$. kangdingensis, $P$. pseudoglauca, $P$.

613 schneideri and P. xiangchengensis was calculated with DnaSP 5.0 software (window length: 600

614 bp, step size 200 bp). X-axis: position of the midpoint of a window, Y-axis: nucleotide diversity

615 of each window.

616 Figure 8 Predicted hairpin loops of inversions in the five plastomes of Populus. The

617 structures of hairpin loops in the of the (A) $c c s A-n d h D$, (B) $n d h C-t r n V$, (C) $n d h D-p s a C$, (D)

$618 n d h F-t r n L,(\mathrm{E})$ petA-psbJ and (F) $\operatorname{trn} N$-trnR regions were drawn with RNAstructure. The arrows

619 in the figure indicate the break points in inversion events.

620 Figure $9 \mathrm{Ka} / \mathrm{Ks}$ values of 19 protein-coding genes of the four comparative combinations. Ka:

621 nonsynonymous, Ks: synonymous.

622 Figure 10 Comparison of long repeats among five Populus plastomes. (A) Number of each

623 repeat type; F: forward repeats, P: palindromic repeats, R: reverse repeats and C: complement

624 repeats (B) Frequency of each repeat type by length.

625 Figure 11 Molecular phylogenetic tree of 27 species in the family Salicaceae inferred from

626 ML analyses based on the complete plastome sequence. The numbers associated with each

627 node are bootstrap support values.

628 


\section{Supporting Information}

630 Supplementary file1

631 The raw data from the four Populus species P. cathayana, P. kangdingensis, P. pseudoglauca

632 and P. schneideri.

633

634 Table S1 GenBank accession numbers of Populus, Salix and outgroups with cp genome

635 sequences used for phylogenetic analyses

636 Table S2 List of genes in the cp genomes

637 Table S3 Codon usage in the five Populus plastomes

638 Table S4 Transitions (Ts) and transversions (Tv) in the four plastomes relative to the plastome 639 of $P$. xiangchengensis

640 Table S5 Synonymous (Ks) and nonsynonymous $(\mathrm{Ka})$ analysis of the five species based on 78

641 unique CDS genes, with $P$. xiangchengensis as the reference

642 Table S6 SSRs in the five cp genomes

643 Table $\mathbf{S 7}$ Repeat sequences in the five cp genomes

644

645

646 Figure S1 Molecular phylogenetics tree of 27 species in the family Salicaceae inferred from ML

647 analyses based on LSC regions. The numbers associated with each node are bootstrap support

648 values.

649 Figure S2 Molecular phylogenetics tree of 27 species in the family Salicaceae inferred from ML

650 analyses based on SSC regions. The numbers associated with each node are bootstrap support

651 values.

652 Figure S3 Molecular phylogenetics tree of 27 species in the family Salicaceae based on IR

653 regions. The numbers associated with each node are bootstrap support values.

654 Figure S4 Molecular phylogenetics tree of 27 species in the family Salicaceae based on

655 LSC+SSC regions. The numbers associated with each node are bootstrap support values.

656 Figure S5 Molecular phylogenetics tree of 27 species in the family Salicaceae based on

657 LSC+SSC+IR regions. The numbers associated with each node are bootstrap support values.

658 Figure S6 Molecular phylogenetics tree of 27 species in the family Salicaceae based on protein659 coding regions. The numbers associated with each node are bootstrap support values. 
Figure 1

The hybridization schematic diagram of five Populus species.

(A) P. kangdingensis; (B) P. cathayana; (C) P. schneideri; (D) P. pseudoglauca; (E) P.

xiangchengensis. $P$. schneideri $=P$. kangdingensis $\times P$. cathayana, $P$. xiangchengensis $=P$. kangdingensis $\times P$. pseudoglauca, $P$. xiangchengensis $=P$. schneideri $\times$ P. pseudoglauca.

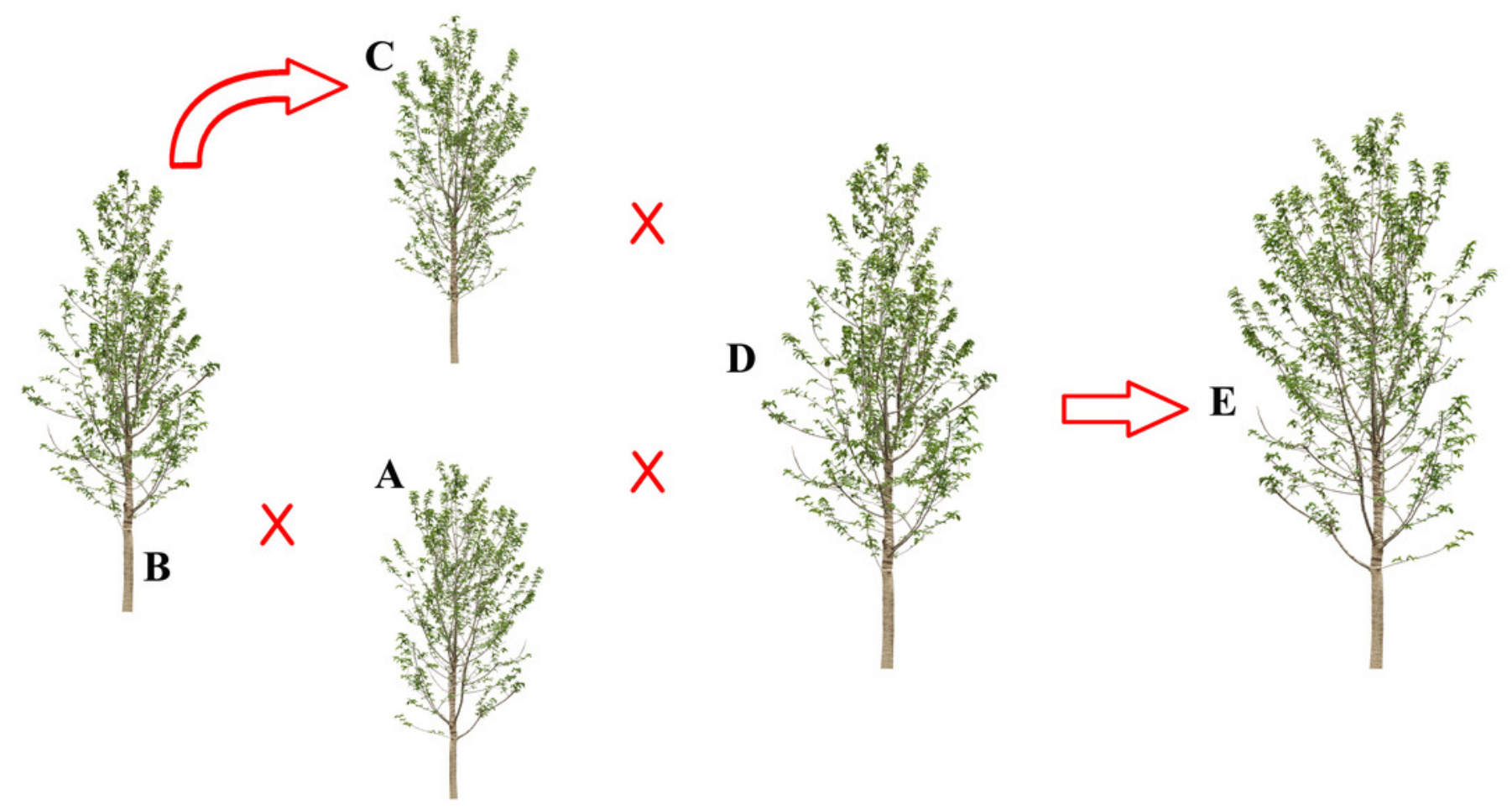


Figure 2

Gene map of the five Populus species cp genomes

The genes that are drawn outside of the circle are transcribed clockwise, whereas those that are drawn inside the circle are transcribed counterclockwise. Large single copy (LSC), small single copy (SSC) and inverted repeats (IRa and IRb) are indicated.

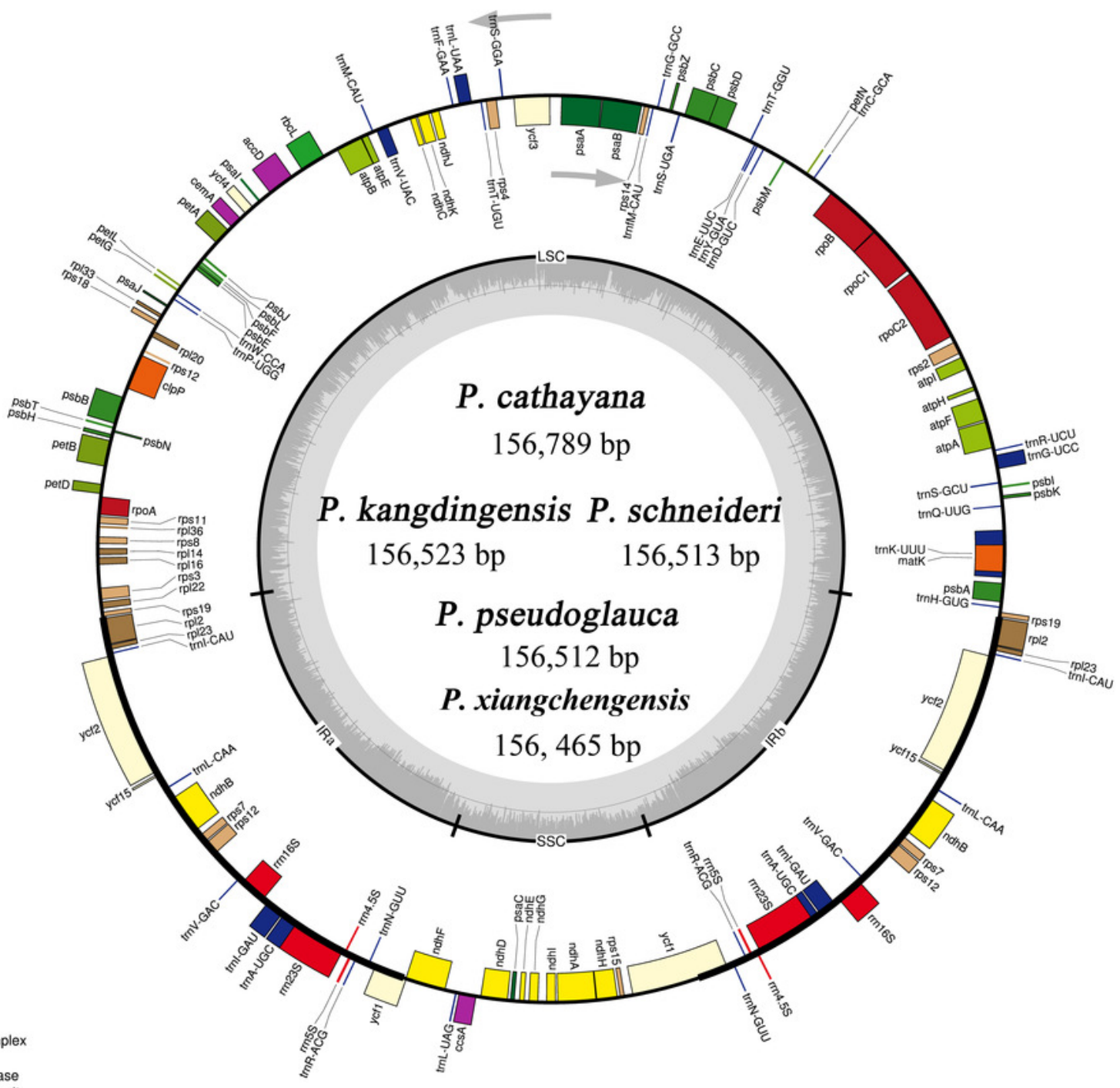

(SSU) 
Figure 3

Amino acid frequencies of the protein-coding sequences of the five plastomes

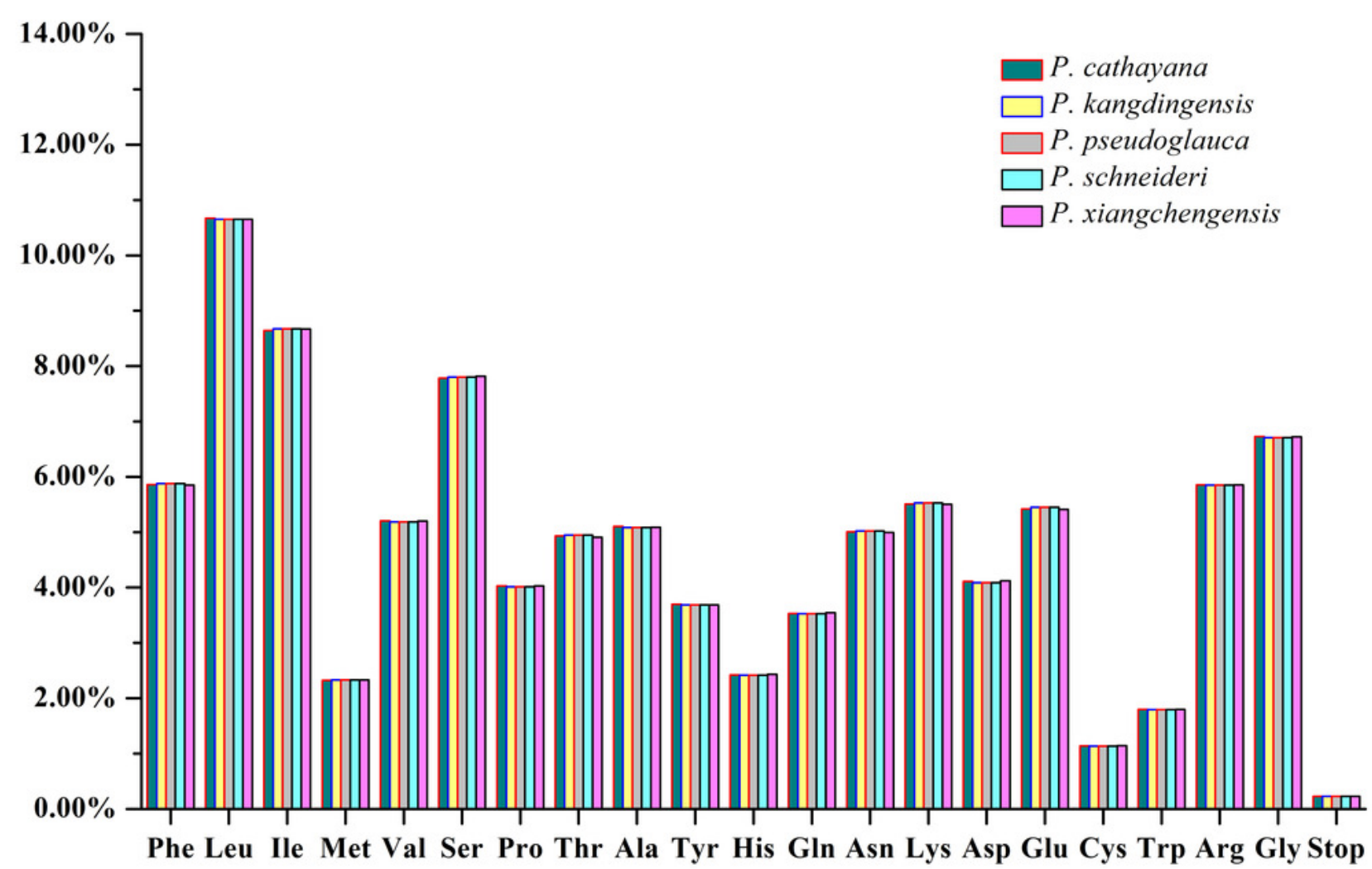




\section{Figure 4}

Whole chloroplast genome alignments for five Populus species using the mVISTA program, with $P$. xiangchengensis as the reference.

The Y-scale indicates identity from $50 \%$ to $100 \%$, Gray arrows indicate the position and direction of each gene. Red indicates non-coding sequences (CNS), blue indicates the exons of protein-coding genes (exon) and line green indicates the tRNA or rRNA genes.

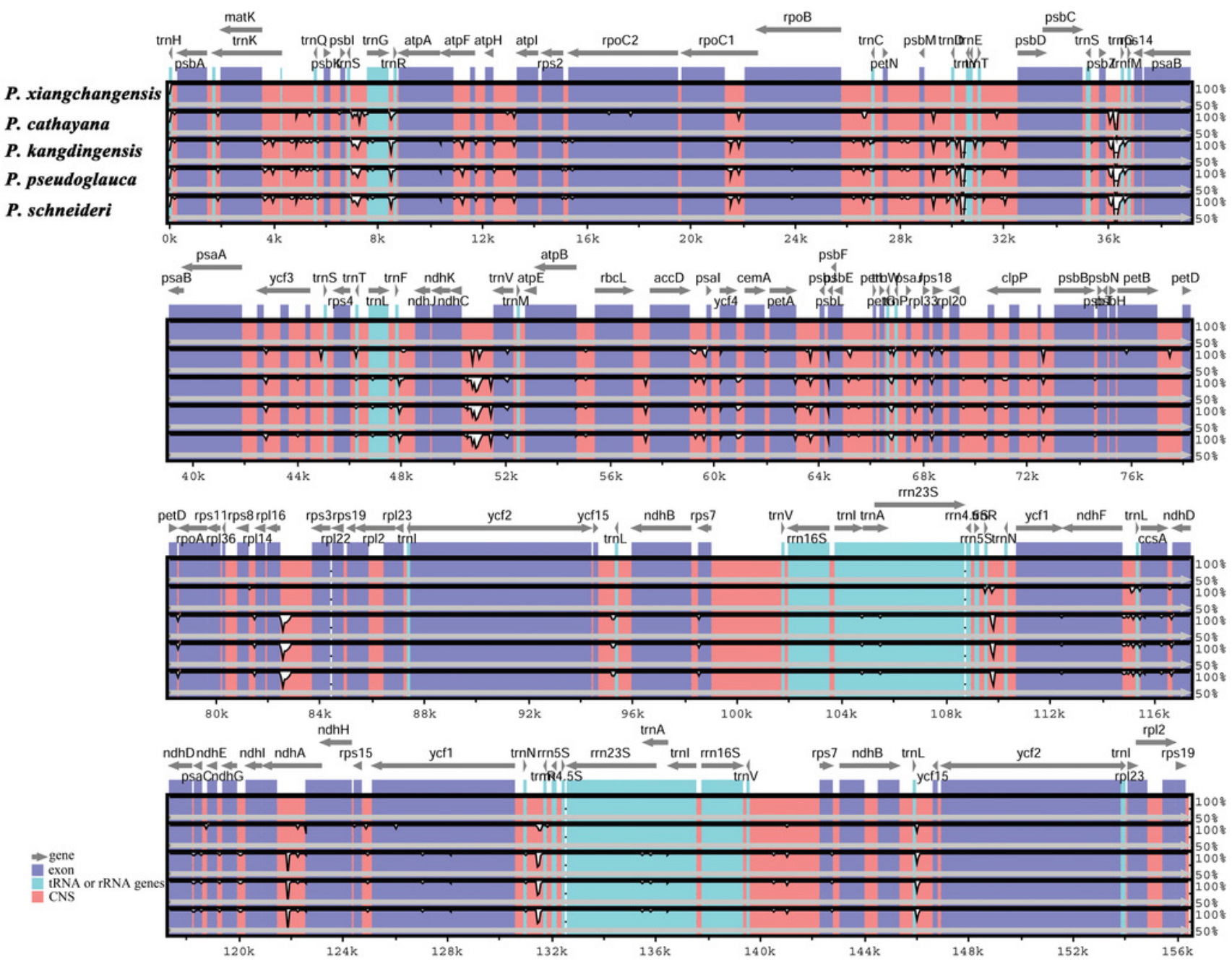


Figure 5

Mauve alignment of the cp genomes of five Populus species

(A) Cp genome of $P$. xiangchengensis; (B) Cp genome of $P$. cathayana; (C) Cp genome of $P$. kangdingensis; (D) Cp genome of P. pseudoglauca; (E) Cp genome of P. schneideri.
A

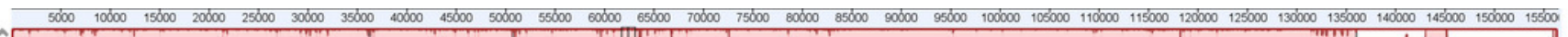

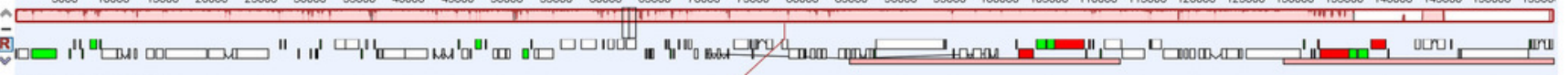

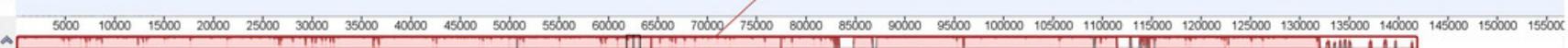
B

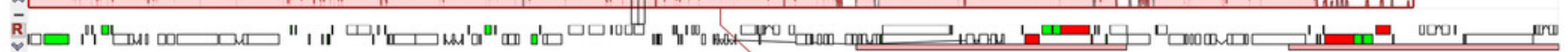

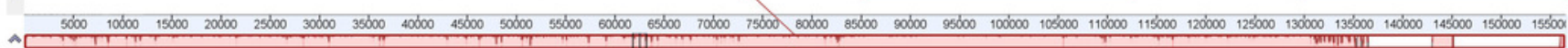
C R

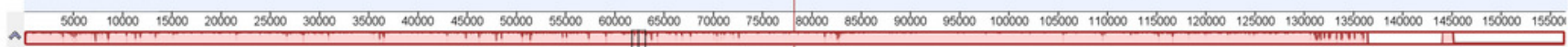
D ${ }_{\text {R }}^{\mathbf{R}}$

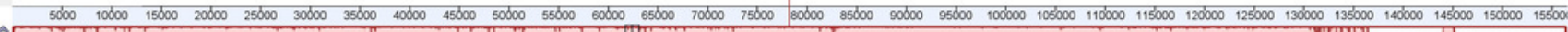
$\mathbf{E}$

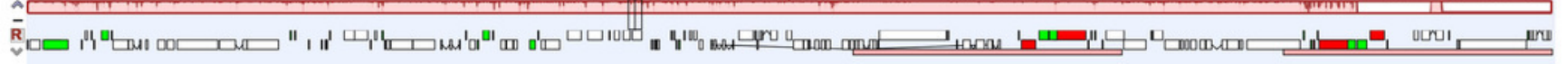


Figure 6

Comparison of LSC, SSC and IR region borders among cp genomes of five Populus species

(A) Cp genome of $P$. xiangchengensis; (B) Cp genome of $P$. cathayana; (C) Cp genome of $P$. kangdingensis; (D) Cp genome of P. pseudoglauca; (E) Cp genome of $P$. schneideri.

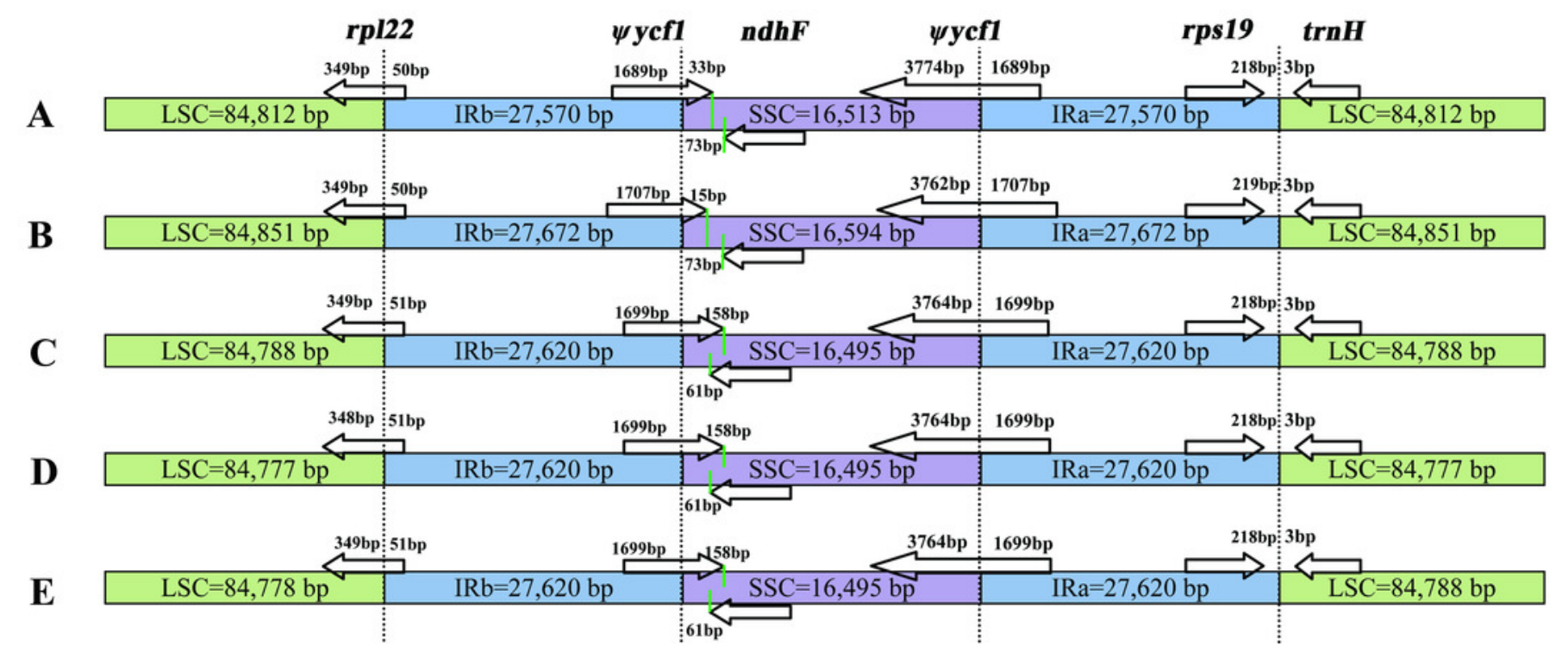


Figure 7

Sliding window analyses of the whole plastomes of five Populus species

The genetic divergence among the plastomes of $P$. cathayana, $P$. kangdingensis, $P$. pseudoglauca, P. schneideri and P. xiangchengensis was calculated with DnaSP 5.0 software (window length: $600 \mathrm{bp}$, step size $200 \mathrm{bp}$ ). X-axis: position of the midpoint of a window, Yaxis: nucleotide diversity of each window.

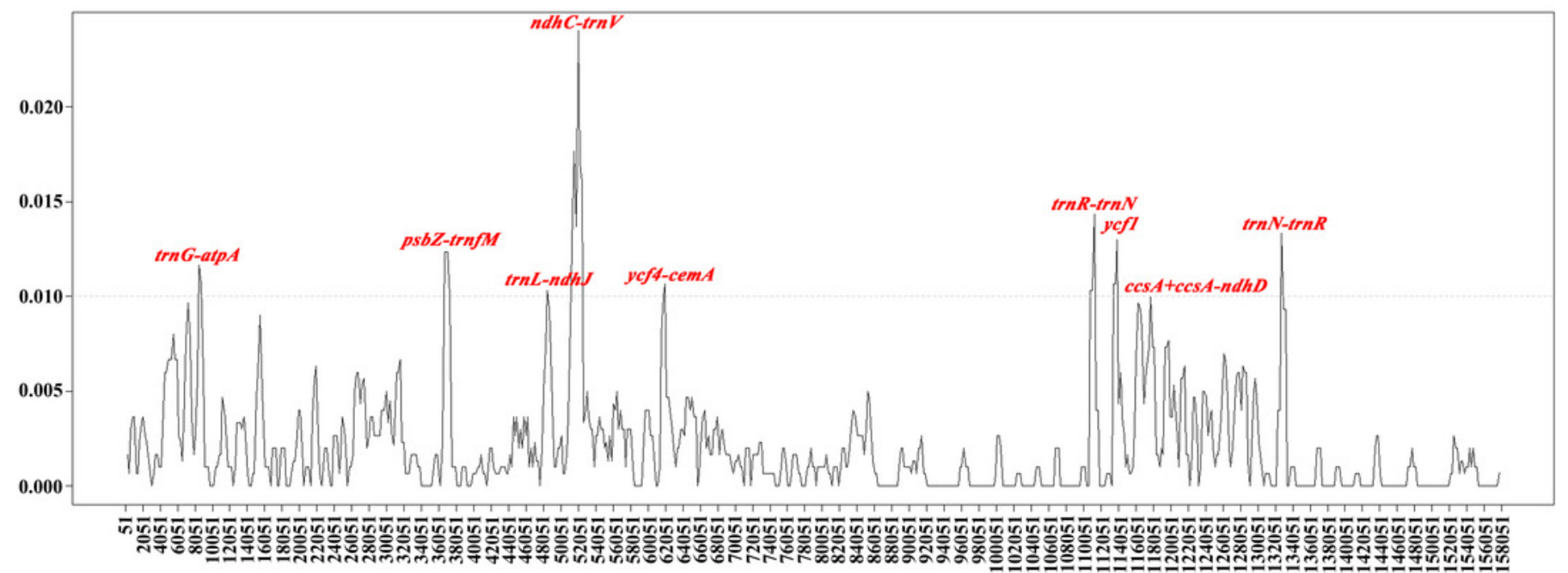


Figure 8

Predicted hairpin loops of inversions in the five plastomes of Populus.

The structures of hairpin loops in the of the (A) ccsA-ndhD, (B) ndhC-trnV, (C) ndhD-psaC, (D) $n d h F-t r n L,(E)$ petA-psbJ and (F) trnN-trnR regions were drawn with RNAstructure. The arrows in the figure indicate the break points in inversion events.

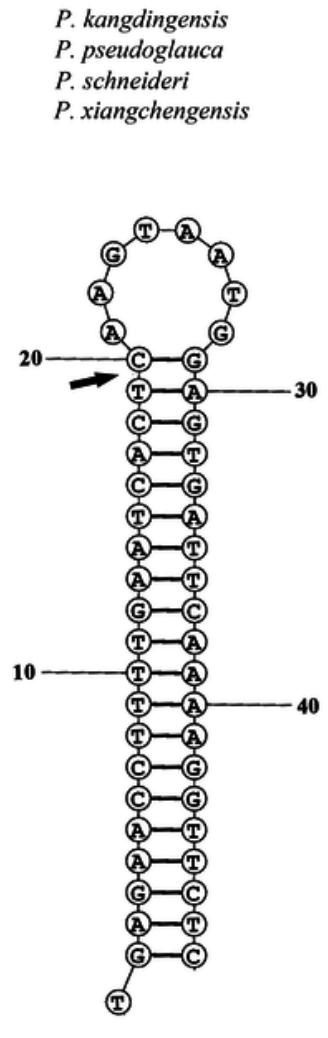

A

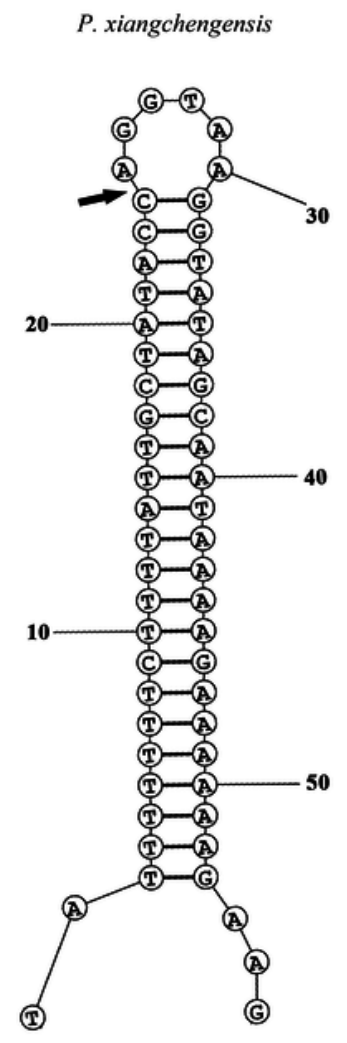

B

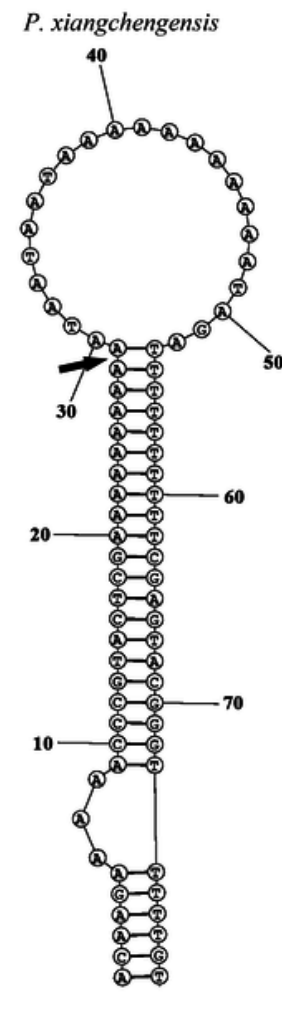

$\mathrm{C}$

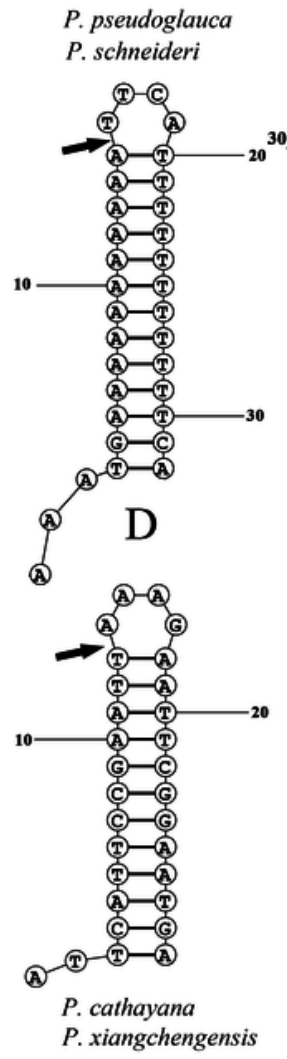

$\mathrm{F}$
P. xiangchengensis

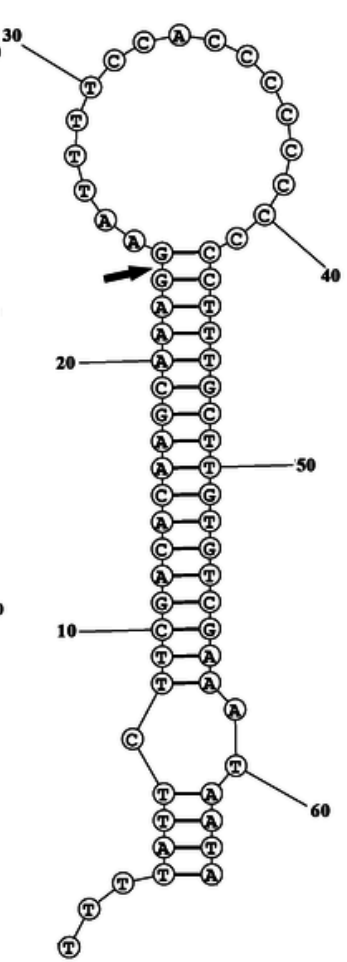

$\mathrm{E}$ 
Figure 9

$\mathrm{Ka} / \mathrm{Ks}$ values of 19 protein-coding genes of the four comparative combinations.

Ka: nonsynonymous, Ks: synonymous.

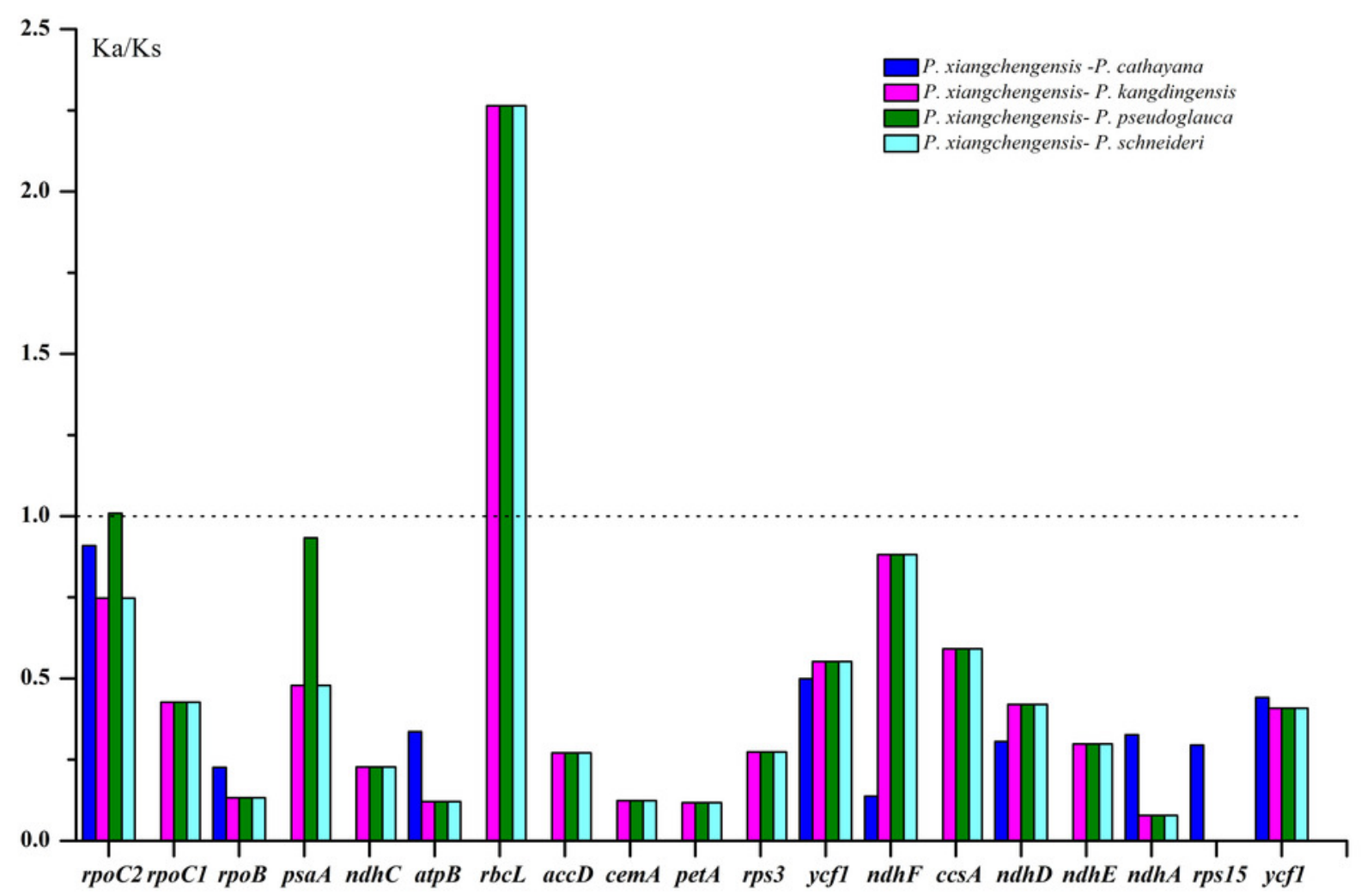


Figure 10

Comparison of long repeats among five Populus plastomes.

(A) Number of each repeat type; F: forward repeats, P: palindromic repeats, R: reverse repeats and C: complement repeats (B) frequency of each repeat type by length.
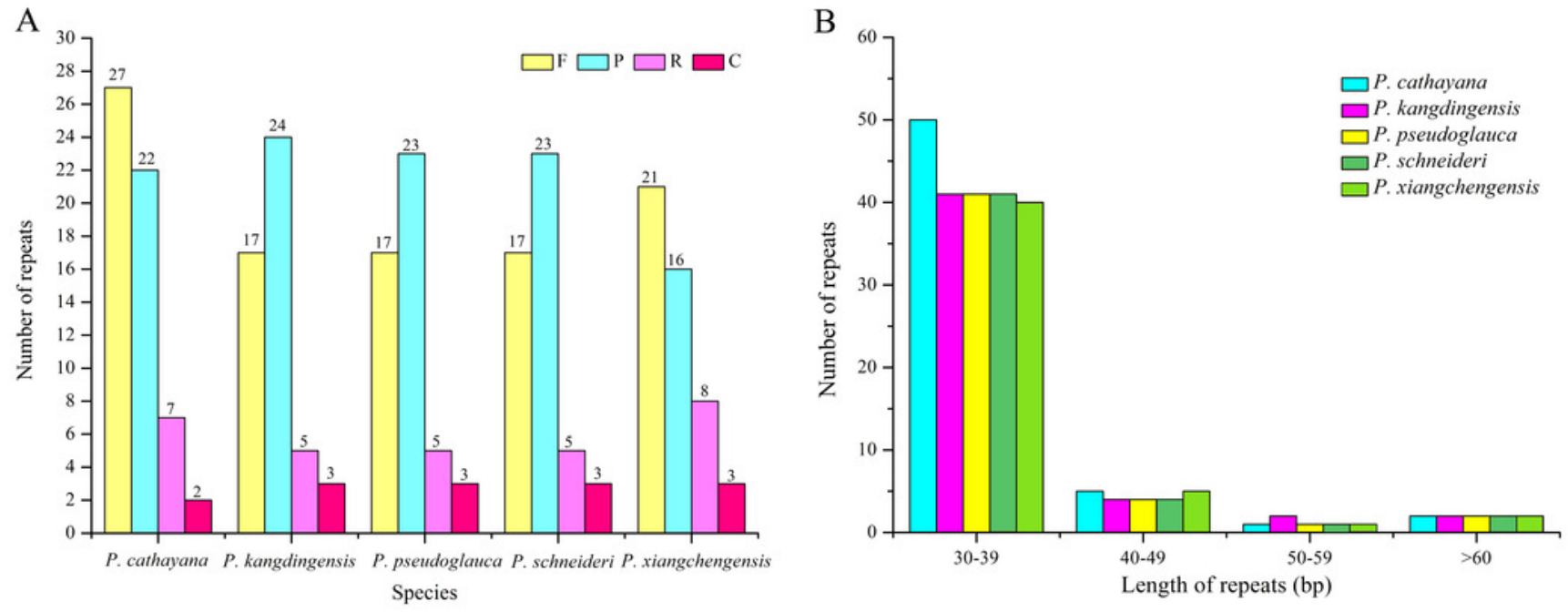


\section{Figure 11}

Molecular phylogenetic tree of 27 species in the family Salicaceae inferred from ML analyses based on the complete plastome sequence.

The numbers associated with each node are bootstrap support values.

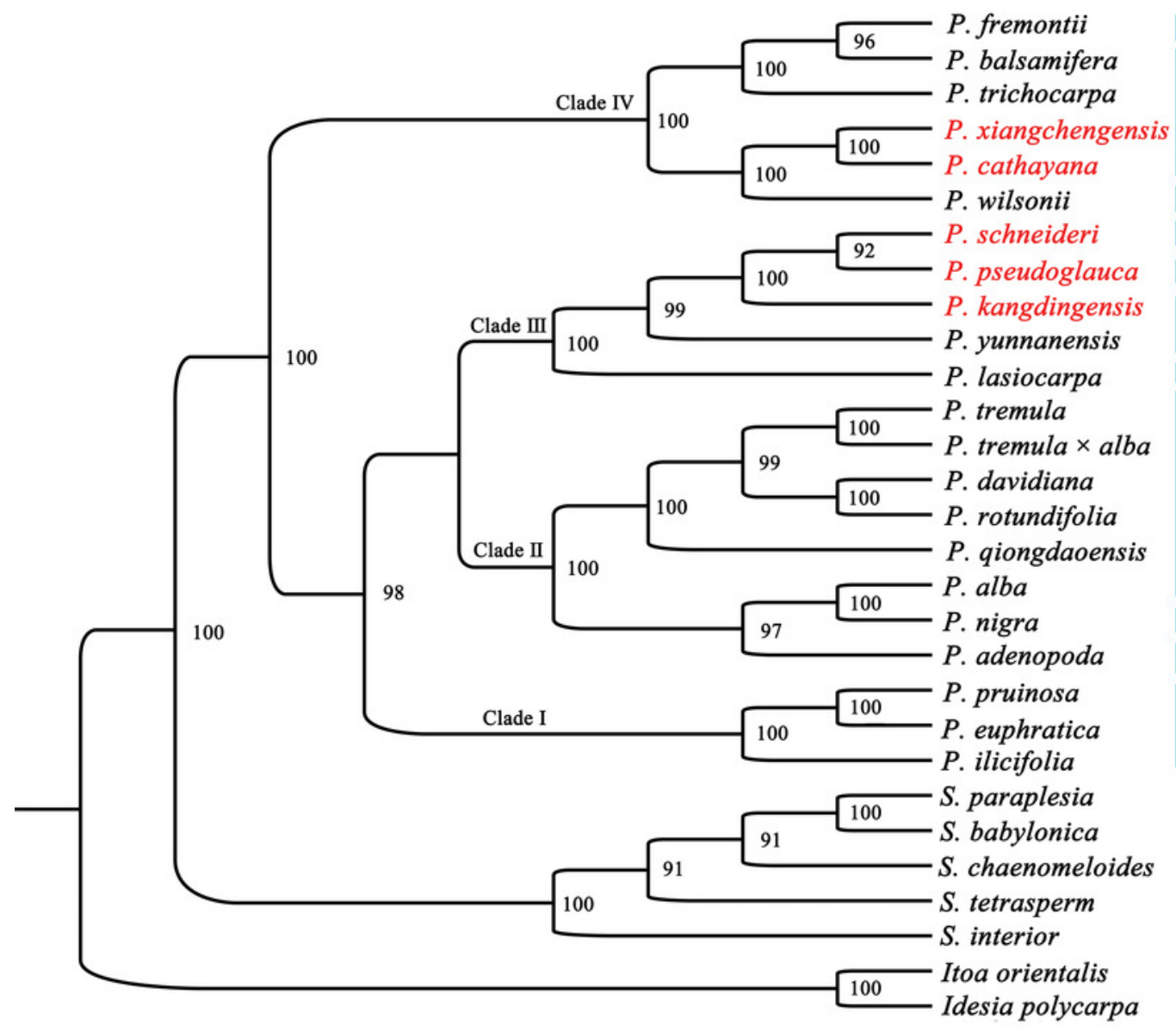

Sec. Aigeiros

Sec. Tacamahaca

Sec. Leucoides

Sec. Tacamahaca

Sec. Leucoides

Sec. Tacamahaca

Sec. Leucoides

Sec. Populus

Sec. Aigeiros

Sec. Populus

Sec. Turanga 


\section{Table $\mathbf{1}$ (on next page)}

The features of five Populus plastomes 
1 Table 1. The features of five Populus plastomes

\begin{tabular}{lllllllll}
\hline Species & Size (bp) & LSC (bp) & SSC(bp) & IR(bp) & $\begin{array}{l}\text { Number of } \\
\text { proteincodi } \\
\text { ng genes }\end{array}$ & $\begin{array}{l}\text { Number of } \\
\text { tRNA genes }\end{array}$ & $\begin{array}{l}\text { Number of } \\
\text { rRNA genes }\end{array}$ & $\begin{array}{l}\text { GC content } \\
(\%)\end{array}$ \\
\hline P. cathayana & 156,789 & 84,851 & 16,594 & 27,672 & $85(7)$ & $37(7)$ & $8(4)$ & 36.7 \\
P. kangdingensis & 156,523 & 84,788 & 16,495 & 27,620 & $85(7)$ & $37(7)$ & $8(4)$ & 36.7 \\
P. pseudoglauca & 156,512 & 84,777 & 16,495 & 27,620 & $85(7)$ & $37(7)$ & $8(4)$ & 36.7 \\
P. schneideri & 156,513 & 84,778 & 16,495 & 27,620 & $85(7)$ & $37(7)$ & $8(4)$ & 36.7 \\
P. xiangchengensis & 156,465 & 84,812 & 16,513 & 27,570 & $85(7)$ & $37(7)$ & $8(4)$ & 36.7 \\
\hline
\end{tabular}

2 
Table 2 (on next page)

Base composition of the five Populus plastomes 
1 Table 2. Base composition of the five Populus plastomes

\begin{tabular}{|c|c|c|c|c|c|c|}
\hline Region & & P. cathayana & P. kangdingensis & P. pseudoglauca & P. schneideri & P. xiangchengensis \\
\hline \multirow{5}{*}{$\operatorname{LSC}(\%)$} & $\mathrm{A}$ & 32.0 & 32.1 & 32.1 & 32.1 & 32.1 \\
\hline & $\mathrm{T}$ & 33.4 & 33.4 & 33.4 & 33.4 & 33.4 \\
\hline & $\mathrm{C}$ & 17.7 & 17.7 & 17.7 & 17.7 & 17.7 \\
\hline & $\mathrm{G}$ & 16.8 & 16.8 & 16.8 & 16.8 & 16.8 \\
\hline & $\mathrm{GC}$ & 34.6 & 34.5 & 34.5 & 34.5 & 34.5 \\
\hline \multirow{5}{*}{$\operatorname{SSC}(\%)$} & $\mathrm{A}$ & 34.9 & 34.9 & 34.9 & 34.9 & 34.9 \\
\hline & $\mathrm{T}$ & 34.5 & 34.6 & 34.6 & 34.6 & 34.3 \\
\hline & $\mathrm{C}$ & 16.1 & 16.1 & 16.1 & 16.1 & 16.1 \\
\hline & $\mathrm{G}$ & 14.6 & 14.4 & 14.4 & 14.4 & 14.6 \\
\hline & $\mathrm{GC}$ & 30.6 & 30.5 & 30.5 & 30.5 & 30.7 \\
\hline \multirow{5}{*}{$\operatorname{IR}(\%)$} & $\mathrm{A}$ & 28.9 & 29.0 & 29.0 & 29.0 & 29.0 \\
\hline & $\mathrm{T}$ & 29.1 & 29.0 & 29.0 & 29.0 & 29.1 \\
\hline & $\mathrm{C}$ & 21.8 & 21.8 & 21.8 & 21.8 & 21.8 \\
\hline & $\mathrm{G}$ & 21.8 & 20.1 & 20.1 & 20.1 & 20.2 \\
\hline & $\mathrm{GC}$ & 41.9 & 42.0 & 42.0 & 42.0 & 42.0 \\
\hline \multirow{5}{*}{ Overall length $(\%)$} & $\mathrm{A}$ & 31.3 & 31.3 & 31.3 & 31.3 & 31.3 \\
\hline & $\mathrm{T}$ & 32.0 & 32.0 & 32.0 & 32.0 & 32.0 \\
\hline & $\mathrm{C}$ & 18.7 & 18.7 & 18.7 & 18.7 & 18.7 \\
\hline & $\mathrm{G}$ & 18.0 & 18.0 & 18.0 & 18.0 & 18.1 \\
\hline & $\mathrm{GC}$ & 36.7 & 36.7 & 36.7 & 36.7 & 36.7 \\
\hline
\end{tabular}


Table 3 (on next page)

Pairwise nucleotide divergences of the five Populus plastomes 
1 Table 3. Pairwise nucleotide divergences of the five Populus plastomes

\begin{tabular}{llllll}
\hline Species & P. cathayana & P. kangdingensis & P. pseudoglauca & P. schneideri & P. xiangchengensis \\
\hline P. cathayana & - & & & & \\
P. kangdingensis & 0.00124 & - & & & \\
P. pseudoglauca & 0.00333 & 0.00326 & - & - & \\
P. schneideri & 0.00335 & 0.00327 & 0.00003 & 0.00002 & - \\
P.xiangchengensis & 0.00333 & 0.00325 & 0.00001 & & \\
\hline
\end{tabular}


Table 4(on next page)

Transitions (Ts) and transversions (Tv) in the protein-coding regions of the four compare combinations with the plastome of $P$. xiangchengensis 
1 Table 4. Transitions ( $\mathrm{Ts}$ ) and transversions (Tv) in the protein-coding regions of the four

2 compare combinations with the plastome of $P$. xiangchengensis

\begin{tabular}{|c|c|c|c|c|c|c|c|}
\hline & \multicolumn{2}{|l|}{ Ts } & \multicolumn{4}{|l|}{$\mathbf{T v}$} & \multirow{2}{*}{ Total } \\
\hline & A-G & $\mathrm{C}-\mathrm{T}$ & $\mathrm{A}-\mathrm{T}$ & $\mathrm{A}-\mathrm{C}$ & T-G & G-C & \\
\hline P. cathayana-P. xiangchengensis & 14 & 19 & 13 & 7 & 9 & 8 & 70 \\
\hline$P$. kangdingensis- $P$. xiangchengensis & 42 & 55 & 12 & 14 & 24 & 13 & 160 \\
\hline P. pseudoglauca-P. xiangchengensis & 44 & 57 & 12 & 15 & 24 & 14 & 166 \\
\hline P. schneideri-P. xiangchengensis & 43 & 56 & 12 & 15 & 24 & 14 & 164 \\
\hline
\end{tabular}

3 
Table 5(on next page)

Statistics of chloroplast SSRs detected in five Populus plastomes

Notes: P1: mononucleotide repeats; P2: dinucleotide repeats; C: compound repeats 
1 Table 5. Statistics of chloroplast SSRs detected in five Populus plastomes

\begin{tabular}{|c|c|c|c|c|c|c|}
\hline SSR type & & P. cathayana & P. kangdingensis & P.pseudoglauca & P. schneideri & P. xiangchengensis \\
\hline \multirow{13}{*}{$\mathrm{P} 1$} & (A) 12 & 3 & 9 & 9 & 9 & 3 \\
\hline & (A) 13 & 5 & 5 & 5 & 5 & 4 \\
\hline & (A) 14 & 2 & 2 & 2 & 2 & 3 \\
\hline & (A) 15 & 2 & 0 & 0 & 0 & 1 \\
\hline & (A) 16 & 1 & 2 & 2 & 2 & 0 \\
\hline & (A) 17 & 0 & 1 & 1 & 1 & 0 \\
\hline & (T) 12 & 4 & 5 & 5 & 5 & 3 \\
\hline & (T) 13 & 3 & 3 & 3 & 3 & 3 \\
\hline & (T) 14 & 3 & 1 & 1 & 1 & 1 \\
\hline & (T) 15 & 1 & 1 & 1 & 1 & 3 \\
\hline & (T) 16 & 2 & 4 & 4 & 4 & 1 \\
\hline & (T) 17 & 0 & 0 & 0 & 0 & 1 \\
\hline & ALL & 26 & 33 & 33 & 33 & 23 \\
\hline $\mathrm{P} 2$ & $\mathrm{TA} / \mathrm{AT}$ & 1 & 2 & 2 & 2 & 1 \\
\hline $\mathrm{C}$ & & 1 & 4 & 4 & 4 & 1 \\
\hline Total & & 28 & 39 & 39 & 39 & 25 \\
\hline
\end{tabular}

2 Notes:

P1: mononucleotide repeats; P2: dinucleotide repeats; C: compound repeats. 